\title{
Influence of Pinching and Potassium Sources on Growth and Flowers Yield of Calendula officinalis
}

\author{
Seham M.A. El-Gamal \\ Department of Medicinal and Aromatic Plants, Horticulture \\ Research Institute, Agricultural Research Centre, Cairo, \\ Egypt.
}

\begin{abstract}
7 HIS study was planned to increase natural colors of Calendula officinalis using natural treatments. Two field experiments were carried out during the two successive seasons of 2010/2011 and 2011/2012, to study the effect of different Potassium sources (Potassium sulphate, $50 \% \quad \mathrm{~K}_{2} \mathrm{SO}_{4}+\mathrm{K}-\mathrm{Mag}, 50 \% \quad \mathrm{~K}_{2} \mathrm{SO}_{4}+$ Salicylic Acid (SA), $50 \% \mathrm{~K}_{2} \mathrm{SO}_{4}+\mathrm{K}-\mathrm{Mag}+\mathrm{SA}$, Flespar $+\mathrm{K}-$ mag or Potassein) and pinching (at 0.0,7.5,10.0 and $12.5 \mathrm{~cm}$ ) on Calendula officinalis. Data indicated that, all Potassium sources had positive effects in increasing growth characters and flower yield. The treatments of 50\% Potassium sulphate with K-Mag and Salicylic Acid were the most effective treatments for growth and carotenoids content of flowers petals. On the other hand, pinching at $10 \mathrm{~cm}$ from the soil surface of the main stem was the best for different growth parameters and flowers yield. The best interaction treatments were treatments of $50 \%$ Potassium sulphate with K-Mag $+\mathrm{SA}$, and pinching at $10 \mathrm{~cm}$ achieved improving growth, flowers yield with $60 \%$ over than the control (average of two seasons), obtaining the highest content of natural pigment and saving 50\% of Potassium sulphate fertilizer and its negative impacts on both human health and environment.
\end{abstract}

Keywords: Calendula officinalis, Potassium, Pinching, Carotenoids.

Calendula (Calendula officinalis L.) is one of the major medicinal plants grown in field plantations, belongs to Asteraceae (Compositae) family. The petals and pollen contain several important constituents such as triterpenoid esters (antiinflammatory), and carotenoids (flavoxanthin and auroxanthin) which are antioxidants and the source of yellow-orange colour (Hamburger et al., 2003 and Bashir et al., 2006). The plant is used as natural colorant in foods. It is also used in pharmaceutical and cosmetic products. The plant is used in folk medicine for antiseptic and anti-inflammatory. In addition, the plant has been used to cure skin disorders and pain, and as a bactericide (Bolderston et al., 2006 and Bielski \& Szwejkowska, 2013) .

Natural colors are gaining considerable attention since several synthetic colorants have given rise to allergic, toxic and carcinogenic effects. Flavonoids have antioxidant activities which play an important role in food preservation and 
human health by combating damage caused by oxidizing agents. Carotenoids are important to humans and other animals as precursors of vitamin A and carotenoid. In addition, they act as antioxidants, immune enhancers, inhibitors of mutagenes is and transformation, inhibitors of premalignant lesions, screening pigments in primate fovea, and nonphotochemical fluorescence quenchers (Castenmiller \& West, 1998, Meda et al., 2005 and Khalid \& Teixeira da Silva 2010).

Potassium is a multifunctional versatile nutrient indispensable for plants. Potassium regulates the phosphorus metabolism in living plant systems and its main physiological function is to conserve and transfer energy during plant metabolic processes e.g. photosynthesis and respiration. This energy, in one form or another, is essential during the growth and development of plants (WinterSluter and Kramer 1977). Potassium is regarded as an indispensable element for crop growth, as it is involved in every metabolic process including activation of several key enzyme systems, carbohydrate metabolism, protein biosynthesis, assimilate translocation and stomatal movement (Marschner, 1995). Yassen et al., 2010 indicated that adding potassium fertilizer significantly increased number of branches, growth and yield in Calendula Officinalis. Singh et al. (2005 and 2007) found that application of potassium to different medicinal plants (lemongrass and rosemary) produced higher herbage compared to control plants.

Salicylic acid (SA) is a phenolic phytohormone that acts as a key regulator of the signaling network in plants under abiotic and biotic stresses. Also, SA exerts stimulatory effects on various physiological processes related to plant growth and development (Pacheco et al., 2013). Stimulation of growth after exposure to SA has been recorded in some plant species, (Shakirova et al., 2003 and Gunes et al., 2007). It can also contribute to stress tolerance by stimulating highly-branched metabolic responses (Horvath et al., 2007). Kovacik et al. (2009) found that treating Matricaria chamomilla plants with SA significantly stimulated growth.

Pinching, the terminal portion of shoots is removed early, emergence of side branches starts earlier and more number of flowers of good quality and uniform size are produced. It is safe alternative treatment which could be a potential substitute to use plant growth retardants application which widely used in recent years for compacting growth and producing more branches. Results of Omar et al., 1997 on Hibiscus sabdariffa L. showed that pinching increased number of branches and flowers. Also, Pushkar and Singh (2012) indicated that pinching increased flower yield of African marigold.

Moreover, human health has received a great attention nowadays. It was documented that chemical fertilizers have a pollutant effect on the soil and plant and in turn, on the human health. Recently, great attention has focused on the possibility to use natural and safety substitution. Therefore, this study was conducted to use different natural sources of potassium fertilizer and pinching as a natural tool for improving growth, flowers yield and flowers carotenoids content of Calendula officinalis.

Egypt. J. Hort. Vol. 42, No.1 (2015) 


\section{Materials and Methods}

Two field experiments were carried out during two successive seasons of 2010/2011 and 2011/2012, in the Exper. Sta. and Lab. of the Vege. Crops and Ornamental Plants Dept. Fac. of Agric., Mansoura Univ., Egypt. Prior to any practices, a composite soil sample was taken from the soil surface $(0-30 \mathrm{~cm})$ of the experimental site, air dried, sieved by $2 \mathrm{~mm}$ sieve and analy zed for physical and chemical properties of soil according to Jackson (1967) and the analysis results are presented in Table 1.

TABLE 1. Physical and chemical characteristics of the soil.

\begin{tabular}{|l|l|l|c|}
\hline \multicolumn{2}{|c|}{ Physical characteristics } & \multicolumn{2}{c|}{ Chemical characteristics } \\
\hline Texture & Loam & $\mathrm{CaCo}_{3}(\%)$ & 0.85 \\
\hline $\begin{array}{l}\text { Coarse sand (\%) } \\
\text { Fine sand (\%) }\end{array}$ & $\begin{array}{l}0.98 \\
24.40\end{array}$ & Organic matter (\%) & 1.17 \\
\hline Clay (\%) & 43.57 & $\mathrm{~N}(\mathrm{ppm})$ & 56 \\
\hline Silt (\%) & 31.05 & $\mathrm{P}(\mathrm{ppm})$ & 2.9 \\
\hline Electrical conductivity (dS.m $\left.{ }^{-1}\right)$ & 0.49 & $\mathrm{~K}(\mathrm{mg} . \mathrm{kg}-1)$ & 589 \\
\hline pH (1:2.5 soil : water) & 8.23 & $\begin{array}{l}\text { Exchangeable sodium percentage } \\
(\%)\end{array}$ & 51.9 \\
\hline $\begin{array}{l}\text { Anion exchange capacity (meq } \\
\text { soil) }\end{array}$ & $100 \mathrm{~g}^{-1}$ & $\begin{array}{l}\text { Cation exchange capacity (meq } 100 \mathrm{~g}^{-1} \\
\text { soil) }\end{array}$ & 0.51 \\
\hline $\mathrm{CO}_{3}^{--}$ & 0.02 & $\mathrm{Ca}^{2+}$ & 0.38 \\
\hline $\mathrm{HCO}_{3}^{-}$ & 0.53 & $\mathrm{Mg}^{2+}$ & 0.05 \\
\hline $\mathrm{CL}^{-}$ & 0.35 & $\mathrm{~K}^{+}$ & 0.41 \\
\hline $\mathrm{SO}_{4}{ }^{--}$ & 0.49 & $\mathrm{Na}^{+}$ & \\
\hline
\end{tabular}

Seeds of Calendula Officinalis L. plants were kindly provided by the Dept. of Medicinal and Aromatic Plants, Ministry of Agriculture, Egypt, and sown at the end of October during both seasons in hills at $40 \mathrm{~cm}$ distance between hills. Thinning for one plant/hill was done 30 days after sowing. Weeds were removed by hand and the irrigation was carried out whenever plants needed. All plots were received recommended doses of $\mathrm{N}(100 \mathrm{~kg} / \mathrm{fed}$. Ammonium sulphate) and $\mathrm{P}$ (150kg/fed. Calcium super phosphate) and farmyard manure according to the recommendations by the Egyptian Ministry of Agriculture.

The experimental unit area (plot) was $6.75 \mathrm{~m}^{2}$ consist of 3 ridges. Each ridge $3 \mathrm{~m}$ long and $0.75 \mathrm{~m}$ width.

\section{Experiment layout}

The experimental design was split plot in randomized complete block design with three replicates. The main plots were assigned for different potassium treatments as follows: 
- Control recommended dose of $\mathrm{K}_{2} \mathrm{SO}_{4}$.

- $50 \%$ of recommended $\mathrm{K}_{2} \mathrm{SO}_{4}+\mathrm{K}-\mathrm{Mag}$.

- $50 \%$ of recommended $\mathrm{K}_{2} \mathrm{SO}_{4}+$ Salicylic acid.

- $50 \%$ of recommended $\mathrm{K}_{2} \mathrm{SO}_{4}+\mathrm{K}-\mathrm{Mag}+$ Salicylic acid

- Flespar + K-Mag .

- $\quad$ Potassein $-\mathrm{N}$.

Potassein-N (contains $8 \% \mathrm{~N}$ and $10 \% \mathrm{P}_{2} \mathrm{O}_{5}$ ) was provided by the General Organization for Agriculture Equalization Fund (G.O.A.E.F.), Ministry of Agriculture, Egypt. K-Mag was provided by Fac. of Agric., Cairo Univ., Egypt. Flespar was provided by Alahram Comp., Cairo, Egypt.

Salicylic acid was dissolved in absolute ethanol then added drop wise to water (ethanol/water: 1/1000, v/v). 45 days after transplanting, SA was applied (at $20 \mathrm{ppm}$ ) on the foliage of calendula plants with a hand sprayer two times in two week intervals.

Different pinching treatments were applied (after 10 weeks from planting) in the sub plots as follows:

- Without pinching (control)

- Pinching at $7.5 \mathrm{~cm}$ from soil surface.

- Pinching at $10.0 \mathrm{~cm}$ from soil surface.

- Pinching at $12.5 \mathrm{~cm}$ from soil surface.

\section{Data recorded}

At maturity (after 105 days from planting) during each growing season six plants/ replicate, were randomly selected for various vegetative data e.g. plant height $(\mathrm{cm})$, branches number/plant and fresh and dry weights (g/plant). Quantitative analysis for NPK and photosynthetic pigments was done. At harvest stage flowers number per plant, flowers fresh and dry weights and total carotenoids were recorded.

\section{Analytical Methods}

NPK: Nitrogen, Phosphorus, and Potassium according to the methods described by Cottenie et al. (1982).

Chlorophyll Determinations: Chlorophyll a, b and total chlorophyll (Ch) were determined in the blade of the third leaf of the plant tip (terminal leaflet) according to the methods described by Saric et al. (1967).

Determination of total carotenoids: Total carotenoids (mg $100 \mathrm{~g}^{-1}$ ) were determined by a modified method of Razmjoo (1997).

\section{Statistical analysis}

The obtained data were subjected to analysis of variances, and the significant differences among treatment means were determined by Duncans' multiple range test at $\mathrm{P}<5 \%$ as published by Duncan (1965).

Egypt. J. Hort. Vol. 42, No.1 (2015) 


\section{Results and Discussions}

Vegetative growth

It is quite clear from the data presented in Tables 2 and 3 that, potassium sources, pinching and their interactions affected significantly plant height, branches number/plant and fresh and dry weights of plants of Calendula officinalis.

In most cases, application of the different potassium sources promoted vegetative growth and resulted in significant increases in the values recorded for the different growth parameters, compared to the control. Maximum growth was resulted from the treatment $\left(0.5 \mathrm{~K}_{2} \mathrm{SO}_{4}+\mathrm{K}-\mathrm{mag}+\mathrm{SA}\right)$, while the minimum growth values were obtained from Potassein treatment.

Similar promotion of vegetative growth (Table 2) was obtained from pinching at the different heights. The favorable pinching height was pinching at $10 \mathrm{~cm}$ compared to the control and other pinching heights in both seasons.

Concerning the combination between the two factors, there was a significant difference between treatments. The plants fertilized with $0.5 \mathrm{~K}_{2} \mathrm{SO}_{4}+\mathrm{K}$-mag + $\mathrm{SA}$ and pinched at $10 \mathrm{~cm}$ resulted in a maximum plant height (68.03 and 71.50), number of branches per plant (13.67 and 14.67), plant fresh weight (769.90 and $783.87 \mathrm{~g} / \mathrm{plant})$ and plant dry weight (141.40 and $144.24 \mathrm{~g} / \mathrm{plant})$ in the two seasons, respectively (Table 3).

Results mentioned above of vegetative growth under K sources might be due to the role of $\mathrm{K}$ in the enhancement and development of plant tissues through the synthesis of simple sugars and starch and also, translocation of carbohydrates and protein synthesis.

The increase in number of branches might be due to the fact that it checked apical dominance and diverted extra energy to the production of more number of branches and thus more leaves (Pushkar and Singh, 2012). In addition, there is evidence of a cross-talk between the SA and auxin signaling pathways during plant vegetative growth (Rivas-San and Plasencia, 2011). The positive effect of SA could be related to the increasing in $\mathrm{CO}_{2}$ as similation and photosynthetic rate (Karlidage et al., 2009). Pacheco et al. (2013) on marigold mentioned that SA exerts stimulatory effects on various physiological processes related to plant growth and development.

These results are in agreement with Hashemabadi et al. (2012) and Yassen et al. (2010) on Calendula officinalis L. and Pal and Ghosh 2010 on African marigold. El-Masry (1996) on peppermint observed that $\mathrm{K}_{2} \mathrm{SO}_{4}(300 \mathrm{~g} / \mathrm{L})$ produced the highest growth. Liu et al., (2008) indicated that adding potassium fertilizer significantly increased number of branches and yield in crops. 
The results are also in similarity with Khobragade et al. (2012) who worked on China aster and reported a positive effect of pinching. Ryagi et al. (2007) on carnation mentioned that pinching increased number of lateral branches per plant.

TABLE 2. Effect of different potassium sources and pinching on vegetative growth characteristics of Calendula officinalis L. during 2010/2011 and 2011/2012.

\begin{tabular}{|c|c|c|c|c|c|c|c|c|}
\hline \multirow{2}{*}{ Treatment } & \multicolumn{2}{|c|}{ Plant height } & \multicolumn{2}{|c|}{ Branches No. } & \multicolumn{2}{|c|}{ Plant F.W } & \multicolumn{2}{|c|}{ Plant D.W } \\
\hline & $\mathbf{1}^{s t}$ & $2^{\text {nd }}$ & $\mathbf{1}^{\text {st }}$ & $2^{\text {nd }}$ & $\mathbf{1}^{s t}$ & $2^{\text {nd }}$ & $\mathbf{1}^{s t}$ & $2^{\text {nd }}$ \\
\hline \multicolumn{9}{|c|}{ Potassium Sources } \\
\hline Control & $\begin{array}{c}52.84 \\
\mathrm{~d}\end{array}$ & $\begin{array}{c}53.95 \\
\mathrm{c}\end{array}$ & $\begin{array}{c}7.83 \\
\mathrm{~d}\end{array}$ & $\begin{array}{c}8.50 \\
\mathrm{~d}\end{array}$ & $\begin{array}{c}411.28 \\
\mathrm{e}\end{array}$ & $\begin{array}{c}414.68 \\
\mathrm{e}\end{array}$ & $\begin{array}{c}78.60 \\
\mathrm{c}\end{array}$ & $\begin{array}{c}80.27 \\
\mathrm{c}\end{array}$ \\
\hline Potassein & $\begin{array}{c}48.23 \\
\mathrm{e}\end{array}$ & $\begin{array}{c}53.73 \\
b\end{array}$ & $\begin{array}{c}7.33 \\
\mathrm{e}\end{array}$ & $\begin{array}{c}8.00 \\
\mathrm{~d}\end{array}$ & $\begin{array}{c}324.78 \\
f\end{array}$ & $\begin{array}{c}333.27 \\
\mathrm{f}\end{array}$ & $\begin{array}{c}61.14 \\
\mathrm{~d}\end{array}$ & $\begin{array}{c}61.99 \\
\mathrm{~d}\end{array}$ \\
\hline $0.5 \mathrm{~K}_{2} \mathrm{SO}_{4}+\mathrm{K}-\mathrm{mag}$ & $\begin{array}{c}57.03 \\
c\end{array}$ & $\begin{array}{c}58.10 \\
b c\end{array}$ & $\begin{array}{c}9.67 \\
b\end{array}$ & $\begin{array}{c}10.33 \\
b\end{array}$ & $\begin{array}{c}453.43 \\
c\end{array}$ & $\begin{array}{c}464.42 \\
c\end{array}$ & $\begin{array}{c}85.68 \\
\text { bc }\end{array}$ & $\begin{array}{c}87.78 \\
b\end{array}$ \\
\hline Felspar + K-mag & $\begin{array}{c}54.58 \\
\mathrm{~d}\end{array}$ & $\begin{array}{c}56.08 \\
\mathrm{bc}\end{array}$ & $\begin{array}{c}8.67 \\
c\end{array}$ & $\begin{array}{c}9.17 \\
\mathrm{c}\end{array}$ & $\begin{array}{c}431.50 \\
\mathrm{~d}\end{array}$ & $\begin{array}{c}433.85 \\
\mathrm{~d}\end{array}$ & $\begin{array}{c}80.69 \\
\text { bc }\end{array}$ & $\begin{array}{c}82.01 \\
c\end{array}$ \\
\hline $\begin{array}{l}0.5 \mathrm{~K}_{2} \mathrm{SO}_{4}+\mathrm{K}-\mathrm{mag} \\
+\mathrm{SA}\end{array}$ & $\begin{array}{c}64.88 \\
a\end{array}$ & $\begin{array}{c}66.92 \\
\mathrm{a}\end{array}$ & $\begin{array}{c}11.17 \\
\mathrm{a}\end{array}$ & $\begin{array}{c}12.00 \\
\mathrm{a}\end{array}$ & $\begin{array}{c}615.93 \\
\mathrm{a}\end{array}$ & $\begin{array}{c}628.71 \\
a\end{array}$ & $\begin{array}{c}118.83 \\
\mathrm{a}\end{array}$ & $\begin{array}{c}116.14 \\
\mathrm{a}\end{array}$ \\
\hline $0.5 \mathrm{~K}_{2} \mathrm{SO}_{4}+\mathrm{SA}$ & $\begin{array}{c}59.17 \\
b\end{array}$ & $\begin{array}{c}60.59 \\
b\end{array}$ & $\begin{array}{c}10.00 \\
b\end{array}$ & $\begin{array}{c}10.58 \\
b\end{array}$ & $\begin{array}{c}482.79 \\
b\end{array}$ & $\begin{array}{c}494.02 \\
\text { b }\end{array}$ & $\begin{array}{c}88.16 \\
b\end{array}$ & $\begin{array}{c}90.31 \\
\mathrm{~b}\end{array}$ \\
\hline \multicolumn{9}{|c|}{ Pinching } \\
\hline 0 & $\begin{array}{c}49.45 \\
\mathrm{~d}\end{array}$ & $\begin{array}{c}53.85 \\
\mathrm{c}\end{array}$ & $\begin{array}{c}6.00 \\
\mathrm{c}\end{array}$ & $\begin{array}{c}6.61 \\
d\end{array}$ & $\begin{array}{c}344.24 \\
\mathrm{~d}\end{array}$ & $\begin{array}{c}350.64 \\
\mathrm{~d}\end{array}$ & $\begin{array}{c}64.41 \\
\mathrm{~d}\end{array}$ & $\begin{array}{c}66.26 \\
\mathrm{~d}\end{array}$ \\
\hline 7.5 & $\begin{array}{c}58.47 \\
b\end{array}$ & $\begin{array}{c}59.82 \\
b\end{array}$ & $\begin{array}{c}9.83 \\
b\end{array}$ & $\begin{array}{c}10.56 \\
b\end{array}$ & $\begin{array}{c}502.52 \\
\mathrm{~b}\end{array}$ & $\begin{array}{c}511.89 \\
\mathrm{~b}\end{array}$ & $\begin{array}{c}94.17 \\
b\end{array}$ & $\begin{array}{c}95.35 \\
b\end{array}$ \\
\hline 10 & $\begin{array}{c}63.98 \\
\mathrm{a}\end{array}$ & $\begin{array}{c}65.41 \\
\mathrm{a}\end{array}$ & $\begin{array}{c}11.22 \\
\mathrm{a}\end{array}$ & $\begin{array}{c}11.94 \\
\mathrm{a}\end{array}$ & $\begin{array}{c}570.55 \\
\mathrm{a}\end{array}$ & $\begin{array}{c}578.01 \\
\mathrm{a}\end{array}$ & $\begin{array}{c}106.00 \\
\mathrm{a}\end{array}$ & $\begin{array}{c}108.15 \\
\mathrm{a}\end{array}$ \\
\hline 12.5 & $\begin{array}{c}52.59 \\
\mathrm{c}\end{array}$ & $\begin{array}{c}53.83 \\
\mathrm{c}\end{array}$ & $\begin{array}{c}9.39 \\
b\end{array}$ & $\begin{array}{c}9.94 \\
\mathrm{c}\end{array}$ & $\begin{array}{c}395.83 \\
\mathrm{c}\end{array}$ & $\begin{array}{c}405.42 \\
c\end{array}$ & $\begin{array}{c}77.50 \\
\mathrm{c}\end{array}$ & $\begin{array}{c}57.93 \\
\mathrm{c}\end{array}$ \\
\hline
\end{tabular}

Values within the same column followed by the same letters are not significantly different, using Duncan's Multiple Range Test at 5\% level. 
INFLUENCE OF PINCHING AND POTASSIUM SOURCES ...

TABLE 3. Effect of interaction between potassium sources and pinching on vegetative growth characteristics of Calendula officinalis L. during 2010/2011 and 2011/2012.

\begin{tabular}{|c|c|c|c|c|c|c|c|c|c|}
\hline \multirow{2}{*}{\multicolumn{2}{|c|}{\begin{tabular}{|c|} 
Treatments \\
Potassium Pinching
\end{tabular}}} & \multicolumn{2}{|c|}{ Plant height } & \multicolumn{2}{|c|}{ Branches NO. } & \multicolumn{2}{|c|}{ F.W. g/plant } & \multicolumn{2}{|c|}{ D.W. g/plant } \\
\hline & & $1^{s t}$ & $2^{\text {nd }}$ & $\mathbf{1}^{s t}$ & $2^{n d}$ & $1^{s t}$ & $2^{n d}$ & $\mathbf{1}^{s t}$ & $2^{n d}$ \\
\hline \multirow[t]{4}{*}{ Control } & 0 & $\begin{array}{c}45.20 \\
\mathrm{jk}\end{array}$ & $\begin{array}{c}46.03 \\
\mathrm{ij} \\
\end{array}$ & $\begin{array}{l}5.33 \\
\mathrm{mn} \\
\end{array}$ & $\begin{array}{c}5.67 \\
1 \\
\end{array}$ & $\begin{array}{c}285.60 \\
\mathrm{jk}\end{array}$ & $\begin{array}{c}293.77 \\
\mathrm{j}\end{array}$ & $\begin{array}{c}55.70 \\
\mathrm{ijk} \\
\end{array}$ & $\begin{array}{c}57.29 \\
1 \mathrm{~m} \\
\end{array}$ \\
\hline & 7.5 & $\begin{array}{c}53.60 \\
\text { gh }\end{array}$ & $\begin{array}{l}55.23 \\
\text { defghij }\end{array}$ & $\begin{array}{c}8.33 \\
\text { hi }\end{array}$ & $\begin{array}{l}9.33 \\
\text { gh }\end{array}$ & $\begin{array}{c}477.40 \\
\mathrm{f}\end{array}$ & $\begin{array}{c}358.90 \\
\mathrm{f}\end{array}$ & $\begin{array}{c}92.15 \\
\text { ef }\end{array}$ & $\begin{array}{c}93.77 \\
\text { ef }\end{array}$ \\
\hline & 10 & $\begin{array}{c}63.17 \\
b c\end{array}$ & $\begin{array}{l}63.83 \\
\text { abcdef }\end{array}$ & $\begin{array}{l}9.67 \\
\text { fg }\end{array}$ & $\begin{array}{c}10.67 \\
\text { ef }\end{array}$ & $\begin{array}{c}541.40 \\
\text { cde }\end{array}$ & $\begin{array}{c}529.73 \\
\text { def }\end{array}$ & $\begin{array}{c}100.45 \\
\text { def }\end{array}$ & $\begin{array}{c}102.25 \\
\text { def }\end{array}$ \\
\hline & 12.5 & $\begin{array}{c}49.40 \\
\mathrm{i} \\
\end{array}$ & $\begin{array}{c}50.70 \\
\text { ghij }\end{array}$ & $\begin{array}{c}8.00 \\
\text { hij } \\
\end{array}$ & $\begin{array}{c}8.33 \\
\mathrm{hi} \\
\end{array}$ & $\begin{array}{c}340.70 \\
\mathrm{hi}\end{array}$ & $\begin{array}{c}349.33 \\
\mathrm{hi}\end{array}$ & $\begin{array}{c}66.11 \\
\text { hij }\end{array}$ & $\begin{array}{c}67.78 \\
\text { hij } \\
\end{array}$ \\
\hline \multirow{4}{*}{ Potassein } & 0 & $\begin{array}{c}41.70 \\
1\end{array}$ & $\begin{array}{c}60.10 \\
\text { abcdefgh }\end{array}$ & $\begin{array}{c}4.67 \\
n\end{array}$ & $\begin{array}{c}5.33 \\
1\end{array}$ & $\begin{array}{c}254.77 \\
\mathrm{k}\end{array}$ & $\begin{array}{c}263.63 \\
\mathrm{~g}\end{array}$ & $\begin{array}{c}48.69 \\
\mathrm{k}\end{array}$ & $\begin{array}{c}50.36 \\
\mathrm{~m}\end{array}$ \\
\hline & 7.5 & $\begin{array}{l}51.37 \\
\mathrm{hi}\end{array}$ & $\begin{array}{l}52.33 \mathrm{f} \\
\text { ghij }\end{array}$ & $\begin{array}{l}7.00 \\
\mathrm{jk}\end{array}$ & $\begin{array}{l}7.67 \\
\mathrm{ij}\end{array}$ & $\begin{array}{c}339.73 \\
\text { hi }\end{array}$ & $\begin{array}{c}348.37 \\
\mathrm{hi}\end{array}$ & $\begin{array}{c}63.49 \\
\text { hijk }\end{array}$ & $\begin{array}{c}62.17 \\
j \mathrm{~km}\end{array}$ \\
\hline & 10 & $\begin{array}{c}56.90 \\
\text { ef }\end{array}$ & $\begin{array}{c}58.47 \\
\text { bcdefghi }\end{array}$ & $\begin{array}{c}9.67 \\
\text { fg }\end{array}$ & $\begin{array}{c}10.67 \\
\text { ef }\end{array}$ & $\begin{array}{c}413.00 \\
\mathrm{~g}\end{array}$ & $\begin{array}{l}422.00 \\
\mathrm{~g}\end{array}$ & $\begin{array}{l}77.24 \\
\mathrm{gh}\end{array}$ & $\begin{array}{c}78.92 \\
\mathrm{~g}\end{array}$ \\
\hline & 12.5 & $\begin{array}{c}42.97 \\
\mathrm{k} 1\end{array}$ & $\begin{array}{c}44.00 \\
\mathrm{j}\end{array}$ & $\begin{array}{c}8.00 \\
\text { hij }\end{array}$ & $\begin{array}{c}8.33 \\
\mathrm{hi}\end{array}$ & $\begin{array}{c}291.63 \\
\mathrm{ijk} \\
\end{array}$ & $\begin{array}{c}299.07 \\
\mathrm{j}\end{array}$ & $\begin{array}{c}55.13 \\
\mathrm{jk}\end{array}$ & $\begin{array}{c}56.53 \\
1 \mathrm{~m} \\
\end{array}$ \\
\hline \multirow{4}{*}{$\begin{array}{l}0.5 \mathrm{~K}_{2} \mathrm{SO}_{4} \\
+\mathrm{K}-\mathrm{mag}\end{array}$} & 0 & $\begin{array}{c}49.93 \\
\mathrm{i}\end{array}$ & $\begin{array}{l}51.67 \\
\text { fghij }\end{array}$ & $\begin{array}{l}6.33 \\
\mathrm{klm}\end{array}$ & $\begin{array}{l}7.00 \\
\mathrm{jk}\end{array}$ & $\begin{array}{c}340.60 \\
\text { hi }\end{array}$ & $\begin{array}{c}352.37 \\
\text { hi }\end{array}$ & $\begin{array}{c}64.28 \\
\text { hijk }\end{array}$ & $\begin{array}{c}66.61 \\
\mathrm{ijk}\end{array}$ \\
\hline & 7.5 & $\begin{array}{c}59.47 \\
\text { de }\end{array}$ & $\begin{array}{c}60.67 \\
\text { abcdefgh }\end{array}$ & $\begin{array}{r}10.67 \\
\text { cdef }\end{array}$ & $\begin{array}{r}11.33 \\
\text { cdef }\end{array}$ & $\begin{array}{c}499.93 \\
\text { def }\end{array}$ & $\begin{array}{c}509.83 \\
\text { ef }\end{array}$ & $\begin{array}{c}94.50 \\
\text { def }\end{array}$ & $\begin{array}{c}96.41 \\
\text { def }\end{array}$ \\
\hline & 10 & $\begin{array}{c}65.33 \\
\mathrm{ab} \\
\end{array}$ & $\begin{array}{c}65.73 \\
\text { abcd }\end{array}$ & $\begin{array}{c}11.67 \\
\text { bcd }\end{array}$ & $\begin{array}{c}12.00 \\
\text { bcd }\end{array}$ & $\begin{array}{c}577.13 \\
\mathrm{c} \\
\end{array}$ & $\begin{array}{c}588.43 \\
\mathrm{c} \\
\end{array}$ & $\begin{array}{c}109.08 \\
\mathrm{~cd}\end{array}$ & $\begin{array}{c}111.17 \\
\mathrm{c}\end{array}$ \\
\hline & 12.5 & $\begin{array}{c}53.40 \\
\text { gh }\end{array}$ & $\begin{array}{l}54.33 \\
\text { defghij }\end{array}$ & $\begin{array}{c}10.00 \\
\text { ef }\end{array}$ & $\begin{array}{c}11.00 \\
\text { def }\end{array}$ & $\begin{array}{c}396.07 \\
\mathrm{~g}\end{array}$ & $\begin{array}{c}407.03 \\
\mathrm{~g}\end{array}$ & $\begin{array}{c}74.87 \\
\text { gh }\end{array}$ & $\begin{array}{c}76.94 \\
\mathrm{gh}\end{array}$ \\
\hline \multirow{4}{*}{$\begin{array}{l}\text { Felspar } \\
\text { K-mag }\end{array}$} & 0 & $\begin{array}{c}46.60 \\
\mathrm{j}\end{array}$ & $\begin{array}{c}48.70 \\
\text { hij }\end{array}$ & $\begin{array}{l}5.67 \\
\mathrm{klm} \\
\end{array}$ & $\begin{array}{c}6.00 \\
\mathrm{kl}\end{array}$ & $\begin{array}{c}320.63 \\
\text { hij }\end{array}$ & $\begin{array}{c}306.67 \\
\mathrm{ij}\end{array}$ & $\begin{array}{c}56.84 \\
\mathrm{ijk} \\
\end{array}$ & $\begin{array}{c}57.98 \\
\mathrm{klm}\end{array}$ \\
\hline & 7.5 & $\begin{array}{c}57.50 \\
\text { ef }\end{array}$ & $\begin{array}{c}58.00 \\
\text { bcdefghi }\end{array}$ & $\begin{array}{c}9.67 \\
\text { fg }\end{array}$ & $\begin{array}{c}10.33 \\
\mathrm{fg}\end{array}$ & $\begin{array}{c}491.07 \\
\text { ef }\end{array}$ & $\begin{array}{l}494.70 \\
\mathrm{f}\end{array}$ & $\begin{array}{c}93.09 \\
\text { ef }\end{array}$ & $\begin{array}{c}93.51 \\
\text { ef }\end{array}$ \\
\hline & 10 & $\begin{array}{c}63.40 \\
\text { bc }\end{array}$ & $\begin{array}{l}65.03 \\
\text { abcde }\end{array}$ & $\begin{array}{r}10.67 \\
\text { cdef }\end{array}$ & $\begin{array}{r}11.33 \\
\text { cdef }\end{array}$ & $\begin{array}{c}544.73 \\
\mathrm{~cd}\end{array}$ & $\begin{array}{c}554.70 \\
\text { cde }\end{array}$ & $\begin{array}{c}102.96 \\
\text { def }\end{array}$ & $\begin{array}{c}104.87 \\
\mathrm{~cd}\end{array}$ \\
\hline & 12.5 & $\begin{array}{l}50.80 \\
\text { hi }\end{array}$ & $\begin{array}{l}52.60 \\
\text { efghij }\end{array}$ & $\begin{array}{l}8.67 \\
\text { fg }\end{array}$ & $\begin{array}{c}9.00 \\
\mathrm{~h}\end{array}$ & $\begin{array}{c}369.57 \\
\text { gh }\end{array}$ & $\begin{array}{c}379.23 \\
\mathrm{gh}\end{array}$ & $\begin{array}{c}69.86 \\
\text { hij }\end{array}$ & $\begin{array}{c}71.69 \\
\text { ghi }\end{array}$ \\
\hline \multirow{4}{*}{$\begin{array}{l}0.5 \mathrm{~K}_{2} \mathrm{SO}_{4} \\
+\mathrm{K}-\mathrm{mag} \\
+\mathrm{SA}\end{array}$} & 0 & $\begin{array}{l}61.20 \\
\mathrm{~cd}\end{array}$ & $\begin{array}{c}62.07 \\
\text { abcdefg }\end{array}$ & $\begin{array}{c}7.33 \\
\mathrm{ijk}\end{array}$ & $\begin{array}{c}8.33 \\
\mathrm{hi}\end{array}$ & $\begin{array}{c}366.37 \\
\mathrm{f}\end{array}$ & $\begin{array}{l}479.70 \\
\mathrm{f}\end{array}$ & $\begin{array}{l}88.20 \\
\text { fg }\end{array}$ & $\begin{array}{c}90.67 \\
\mathrm{f}\end{array}$ \\
\hline & 7.5 & $\begin{array}{c}66.53 \\
a\end{array}$ & $\begin{array}{c}69.00 \\
\mathrm{ab}\end{array}$ & $\begin{array}{c}12.33 \\
b\end{array}$ & $\begin{array}{c}13 . .00 \\
b\end{array}$ & $\begin{array}{c}646.47 \\
b\end{array}$ & $\begin{array}{c}659.80 \\
\mathrm{~b}\end{array}$ & $\begin{array}{c}119.19 \\
b c\end{array}$ & $\begin{array}{c}121.41 \\
\mathrm{~b}\end{array}$ \\
\hline & 10 & $\begin{array}{c}68.03 \\
a\end{array}$ & $\begin{array}{c}71.50 \\
\mathrm{a}\end{array}$ & $\begin{array}{c}13.67 \\
\mathrm{a}\end{array}$ & $\begin{array}{c}14.67 \\
\mathrm{a}\end{array}$ & $\begin{array}{c}769.90 \\
\mathrm{a}\end{array}$ & $\begin{array}{c}783.87 \\
\mathrm{a}\end{array}$ & $\begin{array}{c}141.40 \\
\mathrm{a}\end{array}$ & $\begin{array}{c}144.24 \\
\mathrm{a}\end{array}$ \\
\hline & 12.5 & $\begin{array}{c}63.77 \\
\mathrm{bc}\end{array}$ & $\begin{array}{l}65.10 \\
\text { abcde }\end{array}$ & $\begin{array}{l}11.33 \\
\text { bcde }\end{array}$ & $\begin{array}{c}12.00 \\
\text { bcd }\end{array}$ & $\begin{array}{c}580.97 \\
\mathrm{c} \\
\end{array}$ & $\begin{array}{c}591.47 \\
\mathrm{c}\end{array}$ & $\begin{array}{c}126.52 \\
\mathrm{~b}\end{array}$ & $\begin{array}{c}108.25 \\
\mathrm{c}\end{array}$ \\
\hline \multirow{4}{*}{$\begin{array}{l}0.5 \mathrm{~K}_{2} \mathrm{SO}_{4} \\
+\mathrm{SA}\end{array}$} & 0 & $\begin{array}{c}52.07 \\
\text { hi }\end{array}$ & $\begin{array}{l}54.53 \\
\text { defghij }\end{array}$ & $\begin{array}{c}6.67 \\
\mathrm{k} 1\end{array}$ & $\begin{array}{c}7.33 \\
\mathrm{ij}\end{array}$ & $\begin{array}{c}397.47 \\
\mathrm{~g}\end{array}$ & $\begin{array}{c}407.73 \\
\mathrm{~g}\end{array}$ & $\begin{array}{c}72.75 \\
\mathrm{~h}\end{array}$ & $\begin{array}{c}74.63 \\
\text { ghi }\end{array}$ \\
\hline & 7.5 & $\begin{array}{c}62.33 \\
\mathrm{c}\end{array}$ & $\begin{array}{l}63.67 \\
\text { abcdef }\end{array}$ & $\begin{array}{c}11.00 \\
\text { cdef }\end{array}$ & $\begin{array}{c}11.67 \\
\text { cde }\end{array}$ & $\begin{array}{c}560.50 \\
\mathrm{c}\end{array}$ & $\begin{array}{c}572.73 \\
\text { cd }\end{array}$ & $\begin{array}{c}102.57 \\
\text { bef }\end{array}$ & $\begin{array}{c}104.82 \\
\text { cd }\end{array}$ \\
\hline & 10 & $\begin{array}{c}67.07 \\
\mathrm{a} \\
\end{array}$ & $\begin{array}{c}67.90 \\
a b c \\
\end{array}$ & $\begin{array}{c}12.00 \\
\mathrm{bc}\end{array}$ & $\begin{array}{c}12.33 \\
\mathrm{bc}\end{array}$ & $\begin{array}{c}755.13 \\
\mathrm{c} \\
\end{array}$ & $\begin{array}{c}589.23 \\
\mathrm{c} \\
\end{array}$ & $\begin{array}{c}104.84 \\
\text { cde }\end{array}$ & $\begin{array}{c}107.44 \\
\mathrm{c}\end{array}$ \\
\hline & 12.5 & $\begin{array}{l}55.20 \\
\text { fg }\end{array}$ & $\begin{array}{c}56.27 \\
\text { cdefghij }\end{array}$ & $\begin{array}{c}10.33 \\
\text { def }\end{array}$ & $\begin{array}{c}11.00 \\
\text { def }\end{array}$ & $\begin{array}{c}396.07 \\
\mathrm{~g}\end{array}$ & $\begin{array}{c}406.37 \\
\mathrm{~g}\end{array}$ & $\begin{array}{c}72.49 \\
\text { hi }\end{array}$ & $\begin{array}{c}74.37 \\
\text { ghi }\end{array}$ \\
\hline
\end{tabular}

Values within the same column followed by the same letters are not significantly different, using Duncan's Multiple Range Test at 5\% level.

Egypt. J. Hort. Vol. 42, No.1 (2015) 


\section{Flower yield and contents}

The application of different potassium sources encouraged significantly the production of flowers, flowers fresh and dry weight and carotenoids content in both seasons (Table 4). The most promising effect was found with the treatment by the $0.5 \mathrm{~K}_{2} \mathrm{SO}_{4}+\mathrm{K}$-mag $+\mathrm{SA}$. Since, this treatment gave a great number of flowers per plant (91.00 and 99.42), flowers fresh weight (159.29 and 174.04 $\mathrm{g} /$ plant), flowers dry weight (30.86 and $33.72 \mathrm{~g} / \mathrm{plant}$ ) and total carotenoids (16.20 and $17.93 \mathrm{mg} / 100 \mathrm{~g})$ in the first and second seasons, respectively.

TABLE 4. Effect of different potassium sources and pinching on flowers number, fresh\& dry weight and carotenoids content of Calendula officinalis $\mathrm{L}$. during 2010/2011 and 2011/2012.

\begin{tabular}{|c|c|c|c|c|c|c|c|c|}
\hline \multirow{2}{*}{ Treatment } & \multicolumn{2}{|c|}{ Flowers No. } & \multicolumn{2}{|c|}{ Flowers F.W. } & \multicolumn{2}{|c|}{ Flowers D.W } & \multicolumn{2}{|c|}{$\begin{array}{c}\text { T. } \\
\text { Carotenoids }\end{array}$} \\
\hline & $1^{s t}$ & $2^{n d}$ & $1^{s t}$ & $2^{\text {nd }}$ & $\mathbf{1}^{s t}$ & $2^{n d}$ & $1^{s t}$ & $2^{n d}$ \\
\hline \multicolumn{9}{|c|}{ Potassium Sources } \\
\hline Control & $\begin{array}{c}59.25 \\
\mathrm{e}\end{array}$ & $\begin{array}{c}63.92 \\
\mathrm{e}\end{array}$ & $\begin{array}{c}91.84 \\
\mathrm{e}\end{array}$ & $\begin{array}{c}99.05 \\
\mathrm{e}\end{array}$ & $\begin{array}{c}17.86 \\
\mathrm{~d}\end{array}$ & $\begin{array}{c}19.32 \\
\mathrm{~d}\end{array}$ & $\begin{array}{c}15.48 \\
\mathrm{a}\end{array}$ & $\begin{array}{c}16.66 \\
\mathrm{e}\end{array}$ \\
\hline Potassein & $\begin{array}{c}50.25 \\
\mathrm{f}\end{array}$ & $\begin{array}{c}45.67 \\
\mathrm{f}\end{array}$ & $\begin{array}{c}73.20 \\
\mathrm{f}\end{array}$ & $\begin{array}{c}79.67 \\
\mathrm{f}\end{array}$ & $\begin{array}{c}14.87 \\
\mathrm{e}\end{array}$ & $\begin{array}{c}15.54 \\
\mathrm{e}\end{array}$ & $\begin{array}{c}15.19 \\
\mathrm{a}\end{array}$ & $\begin{array}{c}16.35 \\
\mathrm{f}\end{array}$ \\
\hline $\begin{array}{l}0.5 \mathrm{~K} 2 \mathrm{SO} 4+ \\
\mathrm{K}-\mathrm{mag}\end{array}$ & $\begin{array}{c}69.75 \\
c\end{array}$ & $\begin{array}{c}75.00 \\
\mathrm{c}\end{array}$ & $\begin{array}{c}115.0 \\
9 \mathrm{c}\end{array}$ & $\begin{array}{c}123.7 \\
5 \mathrm{c}\end{array}$ & $\begin{array}{c}19.92 \\
c\end{array}$ & $\begin{array}{c}21.26 \\
c\end{array}$ & $\begin{array}{c}15.00 \\
\mathrm{a}\end{array}$ & $\begin{array}{c}17.64 \\
b\end{array}$ \\
\hline Felspar + K-mag & $\begin{array}{c}64.08 \\
\mathrm{~d}\end{array}$ & $\begin{array}{c}68.57 \\
\mathrm{~d}\end{array}$ & $\begin{array}{c}102.5 \\
7 \mathrm{~d}\end{array}$ & $\begin{array}{c}110.0 \\
5 \mathrm{~d}\end{array}$ & $\begin{array}{c}30.89 \\
\mathrm{a}\end{array}$ & $\begin{array}{c}32.74 \\
\mathrm{a}\end{array}$ & $\begin{array}{c}15.57 \\
\mathrm{a}\end{array}$ & $\begin{array}{c}17.04 \\
\mathrm{~d}\end{array}$ \\
\hline $\begin{array}{l}0.5 \mathrm{~K} 2 \mathrm{SO} 4+ \\
\mathrm{K}-\mathrm{mag}+\mathrm{SA}\end{array}$ & $\begin{array}{c}91.00 \\
\mathrm{a}\end{array}$ & $\begin{array}{c}99.42 \\
\mathrm{a}\end{array}$ & $\begin{array}{c}159.2 \\
9 \mathrm{a}\end{array}$ & $\begin{array}{c}174.0 \\
4 \mathrm{a}\end{array}$ & $\begin{array}{c}30.86 \\
\mathrm{a}\end{array}$ & $\begin{array}{c}33.72 \\
\mathrm{a}\end{array}$ & $\begin{array}{c}16.20 \\
a\end{array}$ & $\begin{array}{c}17.93 \\
\mathrm{a}\end{array}$ \\
\hline $0.5 \mathrm{~K} 2 \mathrm{SO} 4+\mathrm{SA}$ & $\begin{array}{c}76.08 \\
b\end{array}$ & $\begin{array}{c}80.92 \\
b\end{array}$ & $\begin{array}{c}129.3 \\
8 b\end{array}$ & $\begin{array}{c}137.5 \\
8 \mathrm{~b}\end{array}$ & $\begin{array}{c}25.36 \\
b\end{array}$ & $\begin{array}{c}26.52 \\
b\end{array}$ & $\begin{array}{c}15.74 \\
\mathrm{a}\end{array}$ & $\begin{array}{c}17.38 \\
\mathrm{c}\end{array}$ \\
\hline \multicolumn{9}{|c|}{ Pinching } \\
\hline 0 & $\begin{array}{c}52.00 \\
\mathrm{~d}\end{array}$ & $\begin{array}{c}58.98 \\
\mathrm{~d}\end{array}$ & $\begin{array}{c}85.20 \\
\mathrm{~d}\end{array}$ & $\begin{array}{c}96.38 \\
\mathrm{~d}\end{array}$ & $\begin{array}{c}17.37 \\
\mathrm{~d}\end{array}$ & $\begin{array}{c}19.74 \\
\mathrm{~d}\end{array}$ & $\begin{array}{c}13.95 \\
\mathrm{~b}\end{array}$ & $\begin{array}{c}15.19 \\
\mathrm{~d}\end{array}$ \\
\hline 7.5 & $\begin{array}{c}71.83 \\
b\end{array}$ & $\begin{array}{c}76.56 \\
b\end{array}$ & $\begin{array}{c}117.3 \\
9 \mathrm{~b}\end{array}$ & $\begin{array}{c}125.0 \\
9 \mathrm{~b}\end{array}$ & $\begin{array}{c}24.62 \\
b\end{array}$ & $\begin{array}{c}26.12 \\
b\end{array}$ & $\begin{array}{c}16.31 \\
\mathrm{a}\end{array}$ & $\begin{array}{c}17.71 \\
b\end{array}$ \\
\hline 10 & $\begin{array}{c}82.50 \\
\mathrm{a}\end{array}$ & $\begin{array}{c}88.61 \\
\mathrm{a}\end{array}$ & $\begin{array}{c}135.0 \\
2 \mathrm{a}\end{array}$ & $\begin{array}{c}145.0 \\
7 \mathrm{a} \\
\end{array}$ & $\begin{array}{c}28.07 \\
\mathrm{a}\end{array}$ & $\begin{array}{c}30.36 \\
\mathrm{a}\end{array}$ & $\begin{array}{c}17.29 \\
\mathrm{a}\end{array}$ & $\begin{array}{c}19.24 \\
\mathrm{a}\end{array}$ \\
\hline 12.5 & $\begin{array}{c}67.28 \\
\mathrm{c}\end{array}$ & $\begin{array}{c}71.17 \\
\mathrm{c}\end{array}$ & $\begin{array}{c}109.9 \\
6 \mathrm{c}\end{array}$ & $\begin{array}{c}116.2 \\
1 \mathrm{c}\end{array}$ & $\begin{array}{c}22.01 \\
\mathrm{c}\end{array}$ & $\begin{array}{c}23.18 \\
\mathrm{c}\end{array}$ & $\begin{array}{c}14.43 \\
\mathrm{~b}\end{array}$ & $\begin{array}{c}16.54 \\
\mathrm{c}\end{array}$ \\
\hline
\end{tabular}

Values within the same column followed by the same letters are not significantly different, using Duncan's Multiple Range Test at 5\% level.

Similar trend of positive effect was noticed with pinching data presented in the same table. The maximum recorded values were of plants pinched at $10 \mathrm{~cm}$, while the minimum values were of the control plants in the two seasons, respectively.

There was favorable effect of the interaction between potassium sources and pinching. The maximum number of flowers per plant (112.33 and 126), flowers

Egypt. J. Hort. Vol. 42, No.1 (2015) 
fresh weight (196.64 and $220 \mathrm{~g} / \mathrm{plant}$ ), flowers dry weight (38.17 and $42,81 \mathrm{~g} /$ plant $)$ and total carotenoids (17.41 and $20.12 \mathrm{mg} / 100 \mathrm{~g})$ were resulted from the treatment $\left(0.5 \mathrm{~K}_{2} \mathrm{SO}_{4}+\mathrm{K}-\mathrm{mag}+\mathrm{SA}\right.$ and pinching at $\left.10 \mathrm{~cm}\right)$, followed by the treatment $\left(0.5 \mathrm{~K}_{2} \mathrm{SO}_{4}+\mathrm{SA}\right)$ in the first and second seasons, respectively (Table 5). However the plants treated with Potassein without pinching gave the least values in both seasons.

The increase in number of flower, weight of flower and yield of flower per plant under pinching might be due to the fact that it checked apical dominance and diverted extra energy into the production of more number of branches and flowers (Pushkar and Singh, 2012). Moreover, the increase in number of flowers per plant might be attributed to the development of large number of auxiliary shoots as a result of cessation of terminal growth (Gowda and Jaynthi, 1991).

These results are in agreement with those of Bayat et al. (2012) who stated that SA increased flowers number of calendula. Pacheco et al. (2013) on marigold reported that SA application increased biomass accumulation, number of inflorescences and flavonoid content.

Similar results were obtained by Hashemabadi et al. (2012) on Calendula officinalis L., Moradinejad (1995) and Daneshkhah et al. (2007) on rose and Pal and Ghosh (2010) on African marigold. Yassen et al. (2010) on calendula demonstrated that the yield of flowers increased with different sources of potassium. El-Masry (1996) on peppermint found a significant increase in biomass and essential oil yield with $\mathrm{KCl}$ and $\mathrm{K}_{2} \mathrm{SO}_{4}$.

The results are in agreement with those of Pushkar and Singh (2012) who reported that pinching increased flower yield of African marigold. Also, Khobragade et al. (2012) concluded that maximum number of flowers and more weight of flowers per plant in China aster was found significantly higher in the pinched plants than the un-pinched ones.

\section{Chlorophylls and nutrient contents}

Potassium sources significantly induced variable chlorophyll content $(\mathrm{mg} / \mathrm{g})$, nitrogen, phosphorus and potassium percentages in both seasons as presented in Table 6.The $\left(0.5 \mathrm{~K}_{2} \mathrm{SO}_{4}+\mathrm{K}-\mathrm{mag}+\mathrm{SA}\right)$ treatment gave considerably the highest values of total chlorophylls (0.82 and $0.87 \mathrm{mg} / \mathrm{g}), \mathrm{N} \%$ (2.36 and 2.55$), \mathrm{P} \%(0.34$ and 0.37$)$ and $\mathrm{K} \%$ (2.03 and 2.30) in the two seasons, respectively followed by the treatment $\left(0.5 \mathrm{~K}_{2} \mathrm{SO}_{4}+\mathrm{SA}\right)$ in the two seasons, respectively (Table 6).

As shown in the same table different pinching treatments affected significantly on chlorophyll content $(\mathrm{mg} / \mathrm{g})$, nitrogen, phosphorus and potassium percentages in both seasons. The maximum chlorophyll content $(0.86 \mathrm{mg} / \mathrm{g}), \mathrm{N}$ $(0.2 .81 \%), \mathrm{P}(0.39 \%)$ and $\mathrm{K}(2.56 \%)$ were of plants pinched at $10 \mathrm{~cm}$ in the first season. The results of the second season followed the same trend of the first one. 
TABLE 5. Effect of interaction between potassium sources and pinching on flowers number, fresh\& dry weight and carotenoids content of Calendula officinalis L. during 2010/2011 and 2011/2012.

\begin{tabular}{|c|c|c|c|c|c|c|c|c|c|}
\hline \multicolumn{2}{|c|}{ Treatments } & \multicolumn{2}{|c|}{ Flowers No. } & \multicolumn{2}{|c|}{ Flowers F.W. } & \multicolumn{2}{|c|}{ Flowers D.W. } & \multicolumn{2}{|c|}{ Total Carot. } \\
\hline Potassium & Pinching & $\overline{1}^{s t}$ & $2^{n d}$ & $\overline{1}^{s t}$ & $2^{n d}$ & $1^{s t}$ & $2^{n d}$ & $1^{s t}$ & $2^{n d}$ \\
\hline \multirow{4}{*}{ Control } & 0 & $\begin{array}{c}44.33 \\
\text { op }\end{array}$ & $\begin{array}{c}51.00 \\
1\end{array}$ & $\begin{array}{c}68.71 \\
0\end{array}$ & $\begin{array}{c}79.05 \\
\mathrm{~m}\end{array}$ & $\begin{array}{c}13.38 \\
\mathrm{~m}\end{array}$ & $\begin{array}{c}15.41 \\
\mathrm{jk}\end{array}$ & $\begin{array}{c}13.79 \\
\text { efg }\end{array}$ & $\begin{array}{c}14.67 \\
\mathrm{t}\end{array}$ \\
\hline & 7.5 & $\begin{array}{c}62.33 \\
\mathrm{jk}\end{array}$ & $\begin{array}{c}66.33 \\
\mathrm{hi}\end{array}$ & $\begin{array}{c}96.61 \\
\mathrm{kl}\end{array}$ & $\underset{\mathrm{ij}}{102.73}$ & $\underset{\mathrm{ij}}{18.83}$ & $\begin{array}{c}20.05 \\
\text { ghi }\end{array}$ & $\begin{array}{l}15.98 \\
\text { abcdef }\end{array}$ & $\begin{array}{c}17.28 \\
\mathrm{k}\end{array}$ \\
\hline & 10 & $\begin{array}{c}72.33 \\
\text { gh }\end{array}$ & $\begin{array}{c}76.33 \\
\text { ef }\end{array}$ & \begin{tabular}{|c|}
112.12 \\
ghi
\end{tabular} & $\begin{array}{c}118.32 \\
\mathrm{~h}\end{array}$ & $\begin{array}{c}21.83 \\
\mathrm{fg}\end{array}$ & $\begin{array}{c}23.07 \\
\mathrm{fg}\end{array}$ & $\begin{array}{c}17.37 \\
\text { abcd }\end{array}$ & $\begin{array}{c}18.63 \\
\mathrm{e}\end{array}$ \\
\hline & 12.5 & $\begin{array}{c}58.00 \\
\mathrm{kl}\end{array}$ & $\begin{array}{c}62.00 \\
\text { ij }\end{array}$ & 89.90 & $\begin{array}{c}96.10 \\
\mathrm{jk}\end{array}$ & $\begin{array}{c}17.41 \\
\mathrm{jk}\end{array}$ & $\begin{array}{c}18.74 \\
\text { hij }\end{array}$ & $\begin{array}{l}14.81 \\
\text { bcdef }\end{array}$ & $\begin{array}{c}16.07 \\
\mathrm{o}\end{array}$ \\
\hline \multirow{4}{*}{ Potassein } & 0 & $\begin{array}{c}40.67 \\
\mathrm{p}\end{array}$ & $\begin{array}{c}42.33 \\
\mathrm{~m}\end{array}$ & $\begin{array}{c}60.37 \\
\mathrm{~b}\end{array}$ & $\begin{array}{c}62.95 \\
n\end{array}$ & $\underset{n}{11.74}$ & 12.27 & $\begin{array}{c}13.32 \\
\mathrm{fg}\end{array}$ & $\begin{array}{c}14.36 \\
\mathrm{u}\end{array}$ \\
\hline & 7.5 & $\begin{array}{c}53.33 \\
\mathrm{~lm}\end{array}$ & $\begin{array}{c}58.33 \\
\mathrm{jk}\end{array}$ & $\begin{array}{c}77.33 \\
n\end{array}$ & $\begin{array}{c}84.62 \\
\mathrm{~lm}\end{array}$ & $\begin{array}{c}15.08 \\
\mathrm{~lm}\end{array}$ & $\begin{array}{c}16.50 \\
\text { ij }\end{array}$ & $\begin{array}{l}15.80 \\
\text { abcdef }\end{array}$ & $\begin{array}{c}17.06 \\
i\end{array}$ \\
\hline & 10 & 56.33 & $\begin{array}{c}62.67 \\
\text { hij }\end{array}$ & $\begin{array}{c}81.65 \\
\mathrm{mn}\end{array}$ & $\begin{array}{c}90.87 \\
\mathrm{kl}\end{array}$ & $\begin{array}{c}15.92 \\
\mathrm{kl}\end{array}$ & $\begin{array}{c}17.75 \\
\text { hij }\end{array}$ & $\begin{array}{l}17.10 \\
\text { abcde }\end{array}$ & $\begin{array}{c}18.27 \\
\mathrm{~g}\end{array}$ \\
\hline & 12.5 & $\begin{array}{c}50.67 \\
\text { hi }\end{array}$ & $\begin{array}{c}55.33 \\
\mathrm{kl}\end{array}$ & $\begin{array}{c}73.47 \\
\text { no }\end{array}$ & $\begin{array}{c}80.23 \\
\mathrm{~m}\end{array}$ & $\begin{array}{c}14.32 \\
\mathrm{~lm}\end{array}$ & $\begin{array}{c}15.64 \\
\mathrm{jk}\end{array}$ & $\begin{array}{c}14.58 \\
\text { cdef }\end{array}$ & $\begin{array}{c}15.70 \\
\mathrm{q}\end{array}$ \\
\hline \multirow{4}{*}{$\underset{\mathrm{K}-\mathrm{mag}}{0.5 \mathrm{~K} 2 \mathrm{SO} 4}+$} & 0 & $\begin{array}{c}54.00 \\
1 \mathrm{~m}\end{array}$ & 61.33 & $\begin{array}{l}89.10 \\
\mathrm{~lm}\end{array}$ & ${ }_{\mathrm{ij}}^{101.20}$ & $\begin{array}{c}14.36 \\
\mathrm{~lm}\end{array}$ & $\begin{array}{l}16.76 \\
\text { hij }\end{array}$ & $\begin{array}{c}14.13 \\
\text { def }\end{array}$ & $\begin{array}{c}15.70 \\
\mathrm{q}\end{array}$ \\
\hline & 7.5 & $\begin{array}{c}72.00 \\
\text { gh }\end{array}$ & $\begin{array}{c}74.66 \\
\mathrm{f}\end{array}$ & $\begin{array}{c}118.80 \\
\text { fg }\end{array}$ & $\underset{\mathrm{gh}}{123.20}$ & $\begin{array}{c}21.11 \\
\text { fgh }\end{array}$ & $\begin{array}{c}22.59 \\
\text { fg }\end{array}$ & $\begin{array}{l}16.65 \\
\text { abcdef }\end{array}$ & $\begin{array}{c}18.09 \\
\mathrm{~h}\end{array}$ \\
\hline & 10 & $\begin{array}{c}84.33 \\
\mathrm{~d}\end{array}$ & $\begin{array}{c}91.33 \\
\mathrm{c}\end{array}$ & \begin{tabular}{|c|}
139.15 \\
$\mathrm{~d}$
\end{tabular} & $\begin{array}{c}105.70 \\
\mathrm{de}\end{array}$ & $\begin{array}{c}24.73 \\
\mathrm{e}\end{array}$ & $\begin{array}{c}25.32 \\
\mathrm{ef}\end{array}$ & $\begin{array}{c}18.23 \\
\mathrm{a}\end{array}$ & $\begin{array}{c}19.76 \\
\text { b }\end{array}$ \\
\hline & 12.5 & $\begin{array}{c}68.67 \\
\text { hi }\end{array}$ & $\begin{array}{c}72.67 \\
\text { fg }\end{array}$ & $\begin{array}{c}113.30 \\
\text { gh }\end{array}$ & $\begin{array}{c}119.90 \\
\mathrm{~h}\end{array}$ & $\begin{array}{c}19.47 \\
\text { hi }\end{array}$ & $\begin{array}{c}20.36 \\
\text { gh }\end{array}$ & $\begin{array}{c}11.03 \\
\mathrm{~g}\end{array}$ & $\begin{array}{c}17.01 \\
i\end{array}$ \\
\hline \multirow{4}{*}{$\underset{\text { K-mag }}{\text { Felspar }+}$} & 0 & $\begin{array}{c}46.00 \\
\text { no }\end{array}$ & $\begin{array}{c}54.00 \\
\mathrm{kl}\end{array}$ & \begin{tabular}{c|}
73.60 \\
no
\end{tabular} & $\begin{array}{c}86.40 \\
\operatorname{lm}\end{array}$ & 22.18 & $\begin{array}{c}25.87 \\
\text { ef }\end{array}$ & $\begin{array}{c}13.86 \\
\text { efg }\end{array}$ & $\begin{array}{c}15.03 \\
\mathrm{~s}\end{array}$ \\
\hline & 7.5 & $\underset{\mathrm{hi}}{68.00}$ & $\begin{array}{c}72.67 \\
\text { fg }\end{array}$ & \begin{tabular}{|c|}
108.80 \\
$\mathrm{hi}$
\end{tabular} & $\begin{array}{c}116.43 \\
\mathrm{~h}\end{array}$ & $\begin{array}{c}32.94 \\
b\end{array}$ & $\begin{array}{c}34.80 \\
\mathrm{~b}\end{array}$ & $\begin{array}{c}16.25 \\
\text { abcdef }\end{array}$ & 17.51 \\
\hline & 10 & $\begin{array}{c}79.67 \\
\mathrm{de}\end{array}$ & $\begin{array}{c}81.67 \\
\mathrm{de}\end{array}$ & \begin{tabular}{c|}
127.50 \\
$\mathrm{e}$
\end{tabular} & $\begin{array}{c}130.70 \\
\mathrm{fg}\end{array}$ & $\begin{array}{c}38.14 \\
a\end{array}$ & $\begin{array}{c}42.69 \\
a\end{array}$ & $\begin{array}{c}17.73 \\
a b c\end{array}$ & $\begin{array}{c}19.17 \\
\mathrm{e}\end{array}$ \\
\hline & 12.5 & $\begin{array}{c}62.67 \\
\mathrm{jk}\end{array}$ & $\begin{array}{c}66.67 \\
\text { hi }\end{array}$ & $\begin{array}{c}100.37 \\
\mathrm{jk}\end{array}$ & $\underset{\mathrm{i}}{106.67}$ & $\begin{array}{c}30.28 \\
\mathrm{c}\end{array}$ & $\begin{array}{c}27.59 \\
\mathrm{de}\end{array}$ & $\begin{array}{c}15.17 \\
\text { abcdef }\end{array}$ & $\begin{array}{c}16.47 \\
\mathrm{n}\end{array}$ \\
\hline \multirow{4}{*}{$\begin{array}{c}0.5 \mathrm{~K} 2 \mathrm{SO} 4 \\
+\mathrm{K}-\mathrm{Mag} \\
+\mathrm{SA}\end{array}$} & 0 & $\underset{\mathrm{ij}}{65.33}$ & $\begin{array}{c}76.00 \\
\text { ef }\end{array}$ & \begin{tabular}{|c|}
114.33 \\
gh
\end{tabular} & $\begin{array}{c}133.07 \\
\mathrm{f}\end{array}$ & $\underset{f}{22.18}$ & $\begin{array}{c}25.73 \\
\text { ef }\end{array}$ & $\begin{array}{c}14.36 \\
\text { def }\end{array}$ & $\begin{array}{c}15.89 \\
\mathrm{p}\end{array}$ \\
\hline & 7.5 & $\begin{array}{c}97.00 \\
\text { b }\end{array}$ & $\begin{array}{c}102.67 \\
\mathrm{~b}\end{array}$ & $\begin{array}{c}169.80 \\
\mathrm{~b}\end{array}$ & $\begin{array}{c}179.66 \\
\mathrm{~b}\end{array}$ & $\begin{array}{c}32.88 \\
\mathrm{~b}\end{array}$ & $\begin{array}{c}34.80 \\
\text { b }\end{array}$ & $\begin{array}{l}16.74 \\
\text { abcde }\end{array}$ & $\begin{array}{c}18.45 \\
\mathrm{f}\end{array}$ \\
\hline & 10 & $\begin{array}{c}112.33 \\
\mathrm{a}\end{array}$ & $\begin{array}{c}126.00 \\
a\end{array}$ & $\begin{array}{c}196.64 \\
\mathrm{a}\end{array}$ & \begin{tabular}{c|}
220.61 \\
$\mathrm{a}$
\end{tabular} & $\begin{array}{c}38.17 \\
\mathrm{a}\end{array}$ & $\begin{array}{c}42.81 \\
\mathrm{a}\end{array}$ & $\begin{array}{l}17.41 \\
\text { abcdef }\end{array}$ & $\begin{array}{c}20.12 \\
\mathrm{a}\end{array}$ \\
\hline & 12.5 & $\begin{array}{c}89.33 \\
\mathrm{c}\end{array}$ & $\begin{array}{c}93.00 \\
\mathrm{c}\end{array}$ & \begin{tabular}{|c|}
156.40 \\
$\mathrm{c}$
\end{tabular} & $\begin{array}{c}162.82 \\
\mathrm{c}\end{array}$ & $\begin{array}{c}30.19 \\
\mathrm{c}\end{array}$ & $\begin{array}{c}31.54 \\
\text { bc }\end{array}$ & $\begin{array}{l}15.66 \\
\text { abcdef }\end{array}$ & 17.28 \\
\hline \multirow{4}{*}{$\begin{array}{c}0.5 \mathrm{~K} 2 \mathrm{SO} 4 \\
\mathrm{SA}\end{array}$} & 0 & $\begin{array}{c}61_{\mathrm{jk}} 67 \\
{ }^{2}\end{array}$ & $\begin{array}{c}68.00 \\
\text { gh }\end{array}$ & \begin{tabular}{|c|}
105.07 \\
$\mathrm{ij}$
\end{tabular} & $\begin{array}{c}115.60 \\
h\end{array}$ & $\begin{array}{c}20.35 \\
\text { ghi }\end{array}$ & $\begin{array}{l}22.40 \\
\text { fg }\end{array}$ & $\begin{array}{c}14.26 \\
\text { def }\end{array}$ & $\begin{array}{c}15.48 \\
\text { i }\end{array}$ \\
\hline & 7.5 & $\begin{array}{c}78.63 \\
\text { ef }\end{array}$ & $\begin{array}{c}84.67 \\
\mathrm{~d}\end{array}$ & \begin{tabular}{|c|}
$\begin{array}{c}133.03 \\
\mathrm{de}\end{array}$ \\
\end{tabular} & \begin{tabular}{|c|}
143.93 \\
$\mathrm{e}$
\end{tabular} & $\begin{array}{c}26.89 \\
\mathrm{~d}\end{array}$ & $\begin{array}{c}27.97 \\
\text { de }\end{array}$ & $\begin{array}{r}16.47 \\
\text { abcdef }\end{array}$ & $\begin{array}{c}17.87 \\
\mathrm{i}\end{array}$ \\
\hline & 10 & $\begin{array}{c}90.00 \\
\mathrm{c}\end{array}$ & $\underset{c}{93.67}$ & \begin{tabular}{|c|}
153.07 \\
$\mathrm{c}$
\end{tabular} & $\begin{array}{c}159.23 \\
\mathrm{~cd}\end{array}$ & $\underset{c}{29.64}$ & $\begin{array}{c}30.52 \\
\mathrm{~cd}\end{array}$ & $\begin{array}{c}15.96 \\
\mathrm{ab}\end{array}$ & $\begin{array}{c}19.48 \\
\mathrm{c}\end{array}$ \\
\hline & 12.5 & $\begin{array}{c}74.33 \\
\text { fg }\end{array}$ & $\begin{array}{c}77.33 \\
\mathrm{ef}\end{array}$ & $\begin{array}{c}126.37 \\
\mathrm{ef}\end{array}$ & $\begin{array}{c}131.53 \\
\mathrm{ef}\end{array}$ & $\underset{\mathrm{e}}{24.57}$ & $\begin{array}{c}25.18 \\
\text { ef }\end{array}$ & $\begin{array}{l}15.39 \\
\text { abcdef }\end{array}$ & $\begin{array}{c}16.70 \\
\mathrm{~m}\end{array}$ \\
\hline
\end{tabular}

Values within the same column followed by the same letters are not significantly different, using Duncan's Multiple Range Test at 5\% level.

Egypt. J. Hort. Vol. 42, No.1 (2015) 
TABLE 6. Effect of different potassium sources and pinching on total chlorophylls content and N P K percentages of Calendula officinalis L. during 2010/2011 and 2011/2012.

\begin{tabular}{|c|c|c|c|c|c|c|c|c|}
\hline \multirow{2}{*}{ Treatment } & \multicolumn{2}{|c|}{ T. chlorophylls } & \multicolumn{2}{|c|}{$\mathbf{N} \%$} & \multicolumn{2}{|c|}{$\mathbf{P} \%$} & \multicolumn{2}{|c|}{$\mathbf{K} \%$} \\
\hline & $\mathbf{1}^{s t}$ & $2^{\text {nd }}$ & $\mathbf{1}^{s t}$ & $2^{\text {nd }}$ & $\mathbf{1}^{\text {st }}$ & $2^{\text {nd }}$ & $1^{s t}$ & $2^{\text {nd }}$ \\
\hline \multicolumn{9}{|c|}{ Potassium Sources } \\
\hline \multirow{2}{*}{ Control } & 0.67 & 0.82 & 2.17 & 2.33 & 0.36 & 0.35 & 1.77 & 1.9 \\
\hline & d & $\mathrm{e}$ & $\mathrm{e}$ & $\mathrm{e}$ & $\mathrm{a}$ & $\mathrm{d}$ & $\mathrm{cd}$ & $2 \mathrm{e}$ \\
\hline \multirow{2}{*}{ Potassein } & 0.75 & 0.80 & 2.11 & 2.27 & 0.32 & 0.34 & 1.70 & 1.9 \\
\hline & $\mathrm{e}$ & $\mathrm{f}$ & $\mathrm{f}$ & $f$ & $\mathrm{a}$ & $\mathrm{e}$ & d & $8 \mathrm{de}$ \\
\hline \multirow{2}{*}{$0.5 \mathrm{~K} 2 \mathrm{SO} 4+\mathrm{K}-\mathrm{mag}$} & 0.78 & 0.85 & 2.27 & 2.48 & 0.34 & 0.36 & 1.82 & 2.2 \\
\hline & $\mathrm{c}$ & c & $\mathrm{c}$ & $\mathrm{c}$ & $\mathrm{a}$ & $\mathrm{b}$ & $\mathrm{bc}$ & $2 a b$ \\
\hline \multirow{2}{*}{ Felspar + K-mag } & 0.78 & 0.84 & 2.23 & 2.41 & 0.33 & 0.35 & 1.68 & 2.0 \\
\hline & $\mathrm{c}$ & $\mathrm{d}$ & $\mathrm{d}$ & $\mathrm{d}$ & $\mathrm{a}$ & $\mathrm{c}$ & $\mathrm{bc}$ & $7 \mathrm{~cd}$ \\
\hline \multirow{2}{*}{$\begin{array}{l}0.5 \mathrm{~K} 2 \mathrm{SO} 4+\mathrm{K}-\mathrm{mag} \\
+\mathrm{SA}\end{array}$} & 0.82 & 0.87 & 2.36 & 2.55 & 0.34 & 0.37 & 2.03 & 2.3 \\
\hline & $\mathrm{a}$ & $\mathrm{a}$ & $\mathrm{a}$ & $\mathrm{a}$ & $\mathrm{a}$ & $\mathrm{a}$ & $\mathrm{a}$ & $0 \mathrm{a}$ \\
\hline \multirow{2}{*}{$0.5 \mathrm{~K} 2 \mathrm{SO} 4+\mathrm{SA}$} & 0.79 & 0.86 & 2.31 & 2.51 & 0.34 & 0.36 & 1.92 & 2.1 \\
\hline & $\mathrm{b}$ & $\mathrm{b}$ & $\mathrm{b}$ & $\mathrm{b}$ & $\mathrm{a}$ & $\mathrm{b}$ & $\mathrm{b}$ & $5 \mathrm{ab}$ \\
\hline \multicolumn{9}{|c|}{ Pinching } \\
\hline \multirow{2}{*}{0} & 0.72 & 0.78 & 1.86 & 2.05 & 0.30 & 0.30 & 1.23 & 1.4 \\
\hline & $\mathrm{d}$ & $\mathrm{d}$ & $\mathrm{c}$ & $\mathrm{c}$ & b & $\mathrm{d}$ & $\mathrm{d}$ & $0 \mathrm{~d}$ \\
\hline \multirow{2}{*}{7.5} & 0.80 & 0.85 & 2.16 & 2.32 & 0.36 & 0.38 & 1.98 & 2.3 \\
\hline & $\mathrm{b}$ & $\mathrm{b}$ & $\mathrm{b}$ & $\mathrm{b}$ & $\mathrm{a}$ & $\mathrm{b}$ & $\mathrm{b}$ & $0 \mathrm{~b}$ \\
\hline \multirow{2}{*}{10} & 0.86 & 0.89 & 2.81 & 3.00 & 0.39 & 0.41 & 2.56 & 2.9 \\
\hline & $\mathrm{a}$ & $\mathrm{a}$ & $\mathrm{a}$ & $\mathrm{a}$ & $\mathrm{a}$ & $\mathrm{a}$ & $\mathrm{a}$ & $0 \mathrm{a}$ \\
\hline \multirow{2}{*}{12.5} & 0.67 & 0.83 & 2.14 & 2.33 & 0.31 & 0.34 & 1.63 & 1.8 \\
\hline & $\mathrm{c}$ & c & $\mathrm{b}$ & b & $\mathrm{b}$ & $\mathrm{c}$ & $\mathrm{c}$ & $2 c$ \\
\hline
\end{tabular}

Values within the same column followed by the same letters are not significantly different, using Duncan's Multiple Range Test at 5\% level.

Data shown in Table 7 pointed out significant difference between interaction treatments. The plants that received the $\left(0.5 \mathrm{~K}_{2} \mathrm{SO}_{4}+\mathrm{K}-\mathrm{mag}+\mathrm{SA}\right)$ treatment and pinched at $10 \mathrm{~cm}$ gave the highest chlorophyll content $(0.887$ and 0.930 $\mathrm{mg} / \mathrm{g}$ ), N (2.97 and 3.18\%), P (0.399 and 0.417\%) and K (2.69 and 3.09\%), while plants that treated with potassein without pinching had the minimum chlorophyll content $(0.681$ and $0.750 \mathrm{mg} / \mathrm{g}), \mathrm{N}(1.75$ and $1.90 \%), \mathrm{P}(0.266$ and $0.289 \%)$ and $\mathrm{K}(1.07$ and $1.39 \%)$ in the two seasons, respectively.

Generally, nitrogen and phosphorus, contents and uptake increased in the various Potassium sources (Yassen et al., 2010).

Similar results were reported by Bayat et al. (2012) on calendula. They mentioned that SA treated plants had greater chlorophyll reading values than untreated plants. 
TABLE 7. Effect of interaction between potassium sources and pinching on total chlorophylls content and N P K percentages of Calendula officinalis $L$. during 2010/2011 and 2011/2012.

\begin{tabular}{|c|c|c|c|c|c|c|c|c|c|}
\hline \multicolumn{2}{|c|}{ Treatments } & \multicolumn{2}{|c|}{ T. chlorophylls } & \multicolumn{2}{|c|}{ N\% } & \multicolumn{2}{|c|}{$\mathbf{P} \%$} & \multicolumn{2}{|c|}{$\mathbf{K} \%$} \\
\hline Potassium & Pinching & $1^{s t}$ & $2^{\text {nd }}$ & $1^{s t}$ & $2^{n d}$ & $1^{s t}$ & $2^{n d}$ & $1^{\text {st }}$ & $2^{\text {nd }}$ \\
\hline \multirow{4}{*}{ Control } & 0 & $\begin{array}{c}0.694 \\
1\end{array}$ & $\begin{array}{c}0.760 \\
\mathrm{~m}\end{array}$ & $\begin{array}{c}1.78 \\
\mathrm{p}\end{array}$ & $\begin{array}{c}1.95 \\
\mathrm{p}\end{array}$ & $\begin{array}{c}0.373 \\
\text { abcd }\end{array}$ & $\begin{array}{c}0.296 \\
\mathrm{r}\end{array}$ & $\begin{array}{c}1.12 \\
q\end{array}$ & $\begin{array}{c}1.25 \\
\mathrm{n}\end{array}$ \\
\hline & 7.5 & $\begin{array}{c}0.781 \\
\mathrm{gh}\end{array}$ & $\begin{array}{c}0.830 \\
\mathrm{~h}\end{array}$ & $\begin{array}{c}2.09 \\
\mathrm{jk}\end{array}$ & $\begin{array}{c}2.25 \\
\mathrm{k} l\end{array}$ & $\begin{array}{c}0.349 \\
\text { abcd }\end{array}$ & $\begin{array}{c}0.371 \\
\mathrm{i}\end{array}$ & $\begin{array}{c}1.95 \\
\text { fgh }\end{array}$ & $\begin{array}{c}1.89 \\
\mathrm{ij}\end{array}$ \\
\hline & 10 & $\begin{array}{c}0.839 \\
\mathrm{~cd}\end{array}$ & $\begin{array}{c}0.877 \\
\mathrm{e}\end{array}$ & $\begin{array}{c}2.73 \\
\mathrm{~d}\end{array}$ & $\begin{array}{c}2.86 \\
d\end{array}$ & $\begin{array}{c}0.423 \\
\mathrm{a}\end{array}$ & $\begin{array}{c}0.399 \\
d\end{array}$ & $\begin{array}{r}2.46 \\
\text { bcd }\end{array}$ & $\begin{array}{l}2.81 \\
\text { bcd }\end{array}$ \\
\hline & 12.5 & $\begin{array}{c}0.741 \\
\mathrm{jk}\end{array}$ & $\begin{array}{c}0.799 \\
\mathrm{i}\end{array}$ & $\begin{array}{c}2.07 \\
\mathrm{k} 1\end{array}$ & $\begin{array}{c}2.27 \\
\mathrm{ij}\end{array}$ & $\begin{array}{c}0.302 \\
\text { bcd }\end{array}$ & $\begin{array}{c}0.327 \\
\mathrm{~m}\end{array}$ & $\begin{array}{r}1.53 \\
\text { klmn }\end{array}$ & $\begin{array}{l}1.72 \\
\mathrm{ijk}\end{array}$ \\
\hline \multirow{4}{*}{ Potassein } & 0 & $\begin{array}{c}0.681 \\
1\end{array}$ & $\begin{array}{c}0.750 \\
\mathrm{~m}\end{array}$ & $\begin{array}{c}1.75 \\
\mathrm{p}\end{array}$ & $\begin{array}{c}1.90 \\
\mathrm{p}\end{array}$ & $\begin{array}{c}0.266 \\
\mathrm{~d}\end{array}$ & $\begin{array}{c}0.289 \\
\mathrm{~s}\end{array}$ & $\begin{array}{c}1.07 \\
\mathrm{q}\end{array}$ & $\begin{array}{c}1.391 \\
\mathrm{mn}\end{array}$ \\
\hline & 7.5 & $\begin{array}{c}0.766 \\
\text { hij }\end{array}$ & $\begin{array}{c}0.814 \\
\mathrm{i} \\
\end{array}$ & $\begin{array}{c}2.03 \\
1 \\
\end{array}$ & $\begin{array}{c}2.181 \\
\mathrm{~m}\end{array}$ & $\begin{array}{r}0.343 \\
\text { abcd } \\
\end{array}$ & $\begin{array}{c}0.364 \\
\mathrm{j}\end{array}$ & $\begin{array}{r}1.89 \\
\text { ghi } \\
\end{array}$ & $\begin{array}{c}2.15 \\
\text { gh }\end{array}$ \\
\hline & 10 & $\begin{array}{c}0.825 \\
\text { def }\end{array}$ & $\begin{array}{c}0.855 \\
\mathrm{~g} \\
\end{array}$ & $\begin{array}{c}2.61 \\
\mathrm{e}\end{array}$ & $\begin{array}{c}2.79 \\
\mathrm{e}\end{array}$ & $\begin{array}{r}0.371 \\
\text { abcd }\end{array}$ & $\begin{array}{c}0.394 \\
\text { ef }\end{array}$ & $\begin{array}{c}2.38 \\
\mathrm{~cd}\end{array}$ & $\begin{array}{c}2.73 \\
\mathrm{~cd}\end{array}$ \\
\hline & 12.5 & $\begin{array}{c}0.730 \\
\mathrm{k}\end{array}$ & $\begin{array}{c}0.786 \\
\mathrm{kl} \\
\end{array}$ & $\begin{array}{c}2.03 \\
1 \\
\end{array}$ & $\begin{array}{c}2.201 \\
\mathrm{~m}\end{array}$ & $\begin{array}{c}0.295 \\
\text { bcd }\end{array}$ & $\begin{array}{c}0.319 \\
\mathrm{n}\end{array}$ & $\begin{array}{c}1.471 \\
\mathrm{mno}\end{array}$ & $\begin{array}{c}1.65 \\
\mathrm{jkl}\end{array}$ \\
\hline \multirow{4}{*}{$\begin{array}{c}0.5 \mathrm{~K} 2 \mathrm{SO} 4 \\
+\mathrm{K}-\mathrm{mag}\end{array}$} & 0 & $\begin{array}{c}0.729 \\
\mathrm{k}\end{array}$ & $\begin{array}{c}0.790 \\
\mathrm{jk}\end{array}$ & $\begin{array}{c}1.93 \\
\mathrm{n}\end{array}$ & $\begin{array}{c}2.14 \\
\text { no }\end{array}$ & $\begin{array}{c}0.285 \\
\mathrm{~cd}\end{array}$ & $\begin{array}{c}0.310 \\
\text { op }\end{array}$ & $\begin{array}{l}1.34 \\
\text { nop }\end{array}$ & $\begin{array}{l}1.47 \\
\text { klmn }\end{array}$ \\
\hline & 7.5 & $\begin{array}{c}0.820 \\
\text { def }\end{array}$ & $\begin{array}{c}0.870 \\
\mathrm{e}\end{array}$ & $\begin{array}{c}2.23 \\
\mathrm{fg}\end{array}$ & $\begin{array}{c}2.39 \\
\text { gh }\end{array}$ & $\begin{array}{c}0.361 \\
\text { abcd }\end{array}$ & $\begin{array}{c}0.389 \\
\mathrm{f}\end{array}$ & $\begin{array}{c}1.54 \\
\mathrm{k} l \mathrm{mn}\end{array}$ & $\begin{array}{c}2.47 \\
\text { ef }\end{array}$ \\
\hline & 10 & $\begin{array}{c}0.878 \\
\mathrm{ab}\end{array}$ & $\begin{array}{c}0.920 \\
\mathrm{a} \\
\end{array}$ & $\begin{array}{c}2.90 \\
\mathrm{~b}\end{array}$ & $\begin{array}{c}3.13 \\
\mathrm{a} \\
\end{array}$ & $\begin{array}{c}0.393 \\
\mathrm{ab}\end{array}$ & $\begin{array}{c}0.415 \\
\mathrm{ab}\end{array}$ & $\begin{array}{c}2.66 \\
a b\end{array}$ & $\begin{array}{c}3.01 \\
\mathrm{ab}\end{array}$ \\
\hline & 12.5 & $\begin{array}{c}0.772 \\
\mathrm{hi}\end{array}$ & $\begin{array}{c}0.857 \\
\mathrm{f} \\
\end{array}$ & $\begin{array}{c}2.21 \\
\mathrm{fg}\end{array}$ & $\begin{array}{c}2.40 \\
\mathrm{fg}\end{array}$ & $\begin{array}{r}0.321 \\
\text { abcd }\end{array}$ & $\begin{array}{c}0.345 \\
\mathrm{k}\end{array}$ & $\begin{array}{c}1.72 \\
\mathrm{ijk}\end{array}$ & $\begin{array}{c}1.92 \\
\mathrm{hi}\end{array}$ \\
\hline \multirow{4}{*}{$\begin{array}{l}\text { Felspar } \quad+ \\
\text { K-mag }\end{array}$} & 0 & $\begin{array}{c}0.733 \\
\mathrm{k}\end{array}$ & $\begin{array}{c}0.776 \\
1\end{array}$ & $\begin{array}{c}1.85 \\
0\end{array}$ & $\begin{array}{c}2.05 \\
0\end{array}$ & $\begin{array}{c}0.276 \\
\mathrm{~cd}\end{array}$ & $\begin{array}{c}0.305 \\
\mathrm{q}\end{array}$ & $\begin{array}{c}1.19 \\
\mathrm{pq}\end{array}$ & $\begin{array}{c}1.33 \\
\mathrm{mn}\end{array}$ \\
\hline & 7.5 & $\begin{array}{c}0.800 \\
\mathrm{fg}\end{array}$ & $\begin{array}{c}0.843 \\
\mathrm{~g}\end{array}$ & $\begin{array}{c}2.15 \\
\mathrm{hi}\end{array}$ & $\begin{array}{c}2.29 \\
\mathrm{ij} \\
\end{array}$ & $\begin{array}{c}0.353 \\
\text { abcd }\end{array}$ & $\begin{array}{c}0.378 \\
\mathrm{i} \\
\end{array}$ & $\begin{array}{c}2.06 \\
\mathrm{fg}\end{array}$ & $\begin{array}{c}2.31 \\
\mathrm{fg} \\
\end{array}$ \\
\hline & 10 & $\begin{array}{c}0.857 \\
\mathrm{bc}\end{array}$ & $\begin{array}{c}0.894 \\
\mathrm{c} \\
\end{array}$ & $\begin{array}{c}2.81 \\
\mathrm{c}\end{array}$ & $\begin{array}{c}2.97 \\
\mathrm{~d} \\
\end{array}$ & $\begin{array}{c}0.380 \\
\text { abc }\end{array}$ & $\begin{array}{c}0.406 \\
\mathrm{~d}\end{array}$ & $\begin{array}{r}2.57 \\
a b c \\
\end{array}$ & $\begin{array}{c}2.84 \\
a b c\end{array}$ \\
\hline & 12.5 & $\begin{array}{c}0.753 \\
\text { jik }\end{array}$ & $\begin{array}{c}0.823 \\
\mathrm{hi}\end{array}$ & $\begin{array}{c}2.13 \\
\mathrm{ij}\end{array}$ & $\begin{array}{c}2.32 \\
\mathrm{ij} \\
\end{array}$ & $\begin{array}{c}0.311 \\
\text { bcd }\end{array}$ & $\begin{array}{c}0.336 \\
1 \\
\end{array}$ & $\begin{array}{l}1.61 \\
\mathrm{jk} l \mathrm{~m}\end{array}$ & $\begin{array}{c}1.79 \\
\mathrm{ij} \\
\end{array}$ \\
\hline \multirow{4}{*}{$\begin{array}{l}0.5 \mathrm{~K} 2 \mathrm{SO} 4 \\
+\mathrm{K}-\mathrm{mag} \\
+\mathrm{SA}\end{array}$} & 0 & $\begin{array}{c}0.778 \\
\mathrm{gh}\end{array}$ & $\begin{array}{c}0.797 \\
\mathrm{jk} \\
\end{array}$ & $\begin{array}{c}1.98 \\
\mathrm{~m}\end{array}$ & $\begin{array}{c}2.17 \\
\mathrm{mn}\end{array}$ & $\begin{array}{c}0.277 \\
\mathrm{~cd}\end{array}$ & $0.312 \mathrm{o}$ & $\begin{array}{c}1.40 \mathrm{~m} \\
\text { nop }\end{array}$ & $\begin{array}{c}1.53 \mathrm{kl} \\
\mathrm{m}\end{array}$ \\
\hline & 7.5 & $\begin{array}{c}0.826 \\
\text { de }\end{array}$ & $\begin{array}{c}0.884 \\
\mathrm{~cd}\end{array}$ & $\begin{array}{c}2.25 \\
\mathrm{f}\end{array}$ & $\begin{array}{c}2.41 \\
\mathrm{fg} \\
\end{array}$ & $\begin{array}{r}0.365 \\
\text { abcd } \\
\end{array}$ & $\begin{array}{c}0.392 \\
\text { ef }\end{array}$ & $\begin{array}{c}2.27 \\
\mathrm{de} \\
\end{array}$ & $\begin{array}{c}2.58 \\
\mathrm{de}\end{array}$ \\
\hline & 10 & $\begin{array}{c}0.887 \\
\mathrm{a} \\
\end{array}$ & $\begin{array}{c}0.930 \\
\mathrm{a} \\
\end{array}$ & $\begin{array}{c}2.97 \\
\mathrm{a} \\
\end{array}$ & $\begin{array}{c}3.18 \\
\mathrm{a} \\
\end{array}$ & $\begin{array}{c}0.399 \\
\mathrm{ab} \\
\end{array}$ & $\begin{array}{c}0.417 \\
\mathrm{a} \\
\end{array}$ & $\begin{array}{c}2.69 \\
\mathrm{a} \\
\end{array}$ & $\begin{array}{c}3.09 \\
\mathrm{a} \\
\end{array}$ \\
\hline & 12.5 & $\begin{array}{c}0.778 \\
\text { ghi }\end{array}$ & $\begin{array}{c}0.872 \\
\mathrm{e}\end{array}$ & $\begin{array}{c}2.25 \\
\mathrm{f}\end{array}$ & $\begin{array}{c}2.44 \\
\mathrm{f}\end{array}$ & $\begin{array}{c}0.326 \\
\text { abcd }\end{array}$ & $\begin{array}{c}0.351 \\
\mathrm{j}\end{array}$ & $\begin{array}{c}1.79 \\
\text { hij } \\
\end{array}$ & $\begin{array}{c}1.98 \\
\mathrm{hi}\end{array}$ \\
\hline \multirow{4}{*}{$\begin{array}{l}0.5 \mathrm{~K} 2 \mathrm{SO} 4 \\
+\mathrm{SA}\end{array}$} & 0 & $\begin{array}{c}0.687 \\
1\end{array}$ & $\begin{array}{c}0.799 \\
\mathrm{j}\end{array}$ & $\begin{array}{c}1.98 \\
\text { no }\end{array}$ & $\begin{array}{c}2.10 \\
\text { no }\end{array}$ & $\begin{array}{c}0.281 \\
\mathrm{~cd}\end{array}$ & $\begin{array}{c}0.308 \\
\mathrm{pq}\end{array}$ & $\begin{array}{l}1.27 \\
\text { opq }\end{array}$ & $\begin{array}{c}1.411 \\
\mathrm{mn}\end{array}$ \\
\hline & 7.5 & $\begin{array}{c}0.809 \\
\mathrm{fg} \\
\end{array}$ & $\begin{array}{c}0.859 \\
\mathrm{f} \\
\end{array}$ & $\begin{array}{c}2.19 \\
\mathrm{gh}\end{array}$ & $\begin{array}{c}2.39 \\
\mathrm{fg} \\
\end{array}$ & $\begin{array}{r}0.359 \\
\text { abcd } \\
\end{array}$ & $\begin{array}{c}0.385 \\
\mathrm{~g}\end{array}$ & $\begin{array}{c}2.15 \\
\text { ef }\end{array}$ & $\begin{array}{c}2.39 \\
\text { efg }\end{array}$ \\
\hline & 10 & $\begin{array}{c}0.867 \\
a b\end{array}$ & $\begin{array}{c}0.908 \\
\mathrm{~b} \\
\end{array}$ & $\begin{array}{c}2.85 \\
\mathrm{c} \\
\end{array}$ & $\begin{array}{c}3.07 \\
\mathrm{~b}\end{array}$ & $\begin{array}{c}0.397 \\
\mathrm{ab}\end{array}$ & $\begin{array}{c}0.412 \\
\mathrm{~b}\end{array}$ & $\begin{array}{c}2.61 \\
\mathrm{ab} \\
\end{array}$ & $\begin{array}{l}2.93 \\
a b c \\
\end{array}$ \\
\hline & 12.5 & $\begin{array}{c}0.763 \\
\text { hij }\end{array}$ & $\begin{array}{c}0.842 \\
\mathrm{~g}\end{array}$ & $\begin{array}{c}2.15 \\
\text { hi }\end{array}$ & $\begin{array}{c}2.37 \\
\mathrm{gh}\end{array}$ & $\begin{array}{c}0.318 \\
\text { abcd }\end{array}$ & $\begin{array}{c}0.342 \\
\mathrm{k}\end{array}$ & $\begin{array}{c}1.67 \\
\mathrm{jkl}\end{array}$ & $\begin{array}{c}1.86 \\
\mathrm{ij}\end{array}$ \\
\hline
\end{tabular}

Values within the same column followed by the same letters are not significantly different, using Duncan's Multiple Range Test at 5\% level.

Egypt. J. Hort. Vol. 42, No.1 (2015) 
Potassium has been reported to be involved in maximum increase in nutrient uptake (Belorkar et al., 1992) and potassium content in aerial part of Calendula officinalis L. plant (Hashemabadi et al., 2012). Total chlorophyll content in African marigold leaf tissues at active vegetative growing stage showed a gradual increase with increasing potassium level (Pal and Ghosh, 2010).

Acknowledgment : The author thanks Prof. Dr. Mohamed H. El-Masry, Emeritus Professor of Medicinal and Aromatic Plants and Ex-Director of Horticulture Research Institute, Egypt for his valuable suggestions, comments and improvements for the manuscript.

\section{References}

Bashir, S., Janbaz, K.H., Jabeen, Q. and Gilani, A. (2006) Studies on spasmogenic and spasmolytic activities of Calendula officinalis flowers. Phytother. Res., 20, 906-910.

Bayat, H., Alirezaie, M. and Neamati, H. (2012) Impact of exogenous salicylic acid on growth and ornamental characteristics of calendula (Calendula officinalis L.) under salinity stress. J. of Stress Physio. \& Biochem., 8 (1), 259-267.

Belorkar, P.V., Patel, B.N.,Golliwar, V.J. and Kothare, A.J. (1992) Effect of nitrogen and spacing on growth, flowering and yield of African marigold. J. Soils Crops, 2, 62-64.

Bielski, S. and Szwejkowska, B. (2013) Effect of fertilization on the development and yields of pot marigold (Calendula officinalis L.). Herla Polonica, 59 (2), 5-12.

Bolderston, A, Loyd, N.S. and Wong, R.K. (2006) The prevention and management of acute skin reactions related to radiation therapy: a systematic review and practice guideline. Support Care Cancer, 14, 802-817.

Castenmiller, J.J.M. and West, C.E. (1998) Bioavailability and bioconversion of carotenoids. Annu. Rev. Nutr., 18, 19-38.

Cottenie, A., Verloo, M., Kiekens, L., Velghe, G. and Camerlynck, R. (1982) Chemical Analysis of Plant and Soil Laboratory of Analytical and Agrochemistry, State Univ., Ghent, Belgium.

Daneshkhah, M., Kafi, M., Nikbakht, A. and Mirjalili, M.H. (2007) Effects of different levels of nitrogen and potassium fertilizers on flower yield and essential oil content of Rosa damascena Mill. from barzok of Kashan. Iranian J. of Hort. Sci. and Techno. 8 (2), 83-91.

Duncan, D. B. (1965) Multiple Range and Multiple F. Test. Biometrics, 11,1-42.

El-Masry, M.H. (1996) Effect of different sources of potassium on hy droponically grown peppermint (Mentha piperita L.). Menofiya J. Agric. Res., 21(6),1485-1495.

Gowda, N.J.V. and Jayanthi, R. (1991) Effect of cycocel and maleic hydrazide on growth and flowering of African marigold (Tagetes erecta L.), Prog. Hort., 23(1-4), 114-118. 
Gunes, A., Inal, A., Alpaslan, M., Eraslan, F., Guneri Bagci, E. and Cicek, N. (2007) Salicylic acid induced changes on some physiological parameters symptomatic for oxidative stress and mineral nutrition in maize (Zea Mays L.) grown under salinity. J. Plant Physio., 164, 728- 736.

Hamburger, M., Adler, S., Baumann, D., Forg, A. and Weinreich, B. (2003) Preparative purification of the major anti-inflammatory triterpenoid esters from marigold (Calendula officinalis). Fitoterapia, 74, 328-338.

Hashemabadi, D., Mostofipour, A.A., Berimavandi, A.R., Kaviani, B. and Zarchini, M. (2012) Improvement of the yield and essential oils quantitative in Calendula (Calendula officinalis L.) by using different planting arrangement and potassium fertilizer. J. of Ornamental and Horticultural Plants, 2 (3), 147-154.

Horvath, E., Szalai, G. and Janda, T. (2007) Induction of a biotic stress tolerance by Salicylic acid signaling. J. Plant growth Regul., 26, 290 -300 .

Jackson, M.L. (1967) "Soil Chemical Analysis", Printice-Hall of India Private Limited, New Delhi, pp.144-197.

Karlidage, H., Yildirim, E. and Turan, M. (2009) Salicy lic acid ameliorates the adverse effect of salt stress on strawberry. Sci. Agric., 66, 180-187.

Khalid, A. Kh. and Teixeira da Silva, J.A. (2010) Yield, essential oil and pigment content of Calendula officinalis L. flower heads cultivated under salt stress conditions. Scientia Horticulturae, 126, 297-305.

Khobragade, R., Bisen, S. and Thakur, R. (2012) Effect of planting distance and pinching on growth, flowering and yield of China aster (Callistephus chinensis) cv. Poornima. The Indian J. Agric. Sci., 82(4), 334-339.

Kovacik, J., Gruz, J. and Backor, M. (2009) Salicy lic acid - induced changes to growth and phenolic metabolism in Matricaria chamomilla plants. Plant Cell Rep., 28, 135- 143.

Liu, Z.H., Jiang, L.H., Li, X.L., Härdter, R., Zhang, W.J., Zhang, Y.L. and Zheng, D.F. (2008) Effect of $\mathrm{N}$ and $\mathrm{K}$ fertilizers on yield and quality of greenhouse vegetable crops. Pedosphere, 18 (4), 496-502.

Marschner, H. (1995) Mineral Nutrition of Higher Plants $2^{\text {nd }}$ Ed. pp.: 99-101 Academic press, London.

Meda, A., Lamien, C.E., Romito, M., Millogo, J. and Nacoulma, G.O. (2005) Determination of the total phenolic, flavonoid and proline contents in Burkina Fasan honey, as well as their radical scavenging activity. Food Chem., 91, 571-577.

Moradinejad, F. (1995) Effect of $\mathrm{N}$ and $\mathrm{K}$ on quantitative and qualitative parameters of rose. M.Sc. Thesis, Tarbiat Modarres Univ. Tehran. Iran.

Omar, E.A., Khattab, M.E. and Ibrahim, M.E. (1997) Effect of pinching and foliar application of some growth regulators on two new early mature varieties of (Hibiscus sabdariffa L.). Egypt J. Hort., 24 (2), 117-130.

Egypt. J. Hort. Vol. 42, No.1 (2015) 
Pacheco, A.C., Cabral, C.S., Fermino, E.S.S. and Aleman, C.C. (2013) Salicy lic acid - induced changes to growth, flowering and flavonoids production in marigold plants. J. Medicinal Plant Res., 7 (42), 3158 - 3163.

Pal, P. and Ghosh, P. (2010) Effect of different sources and levels of potassium on growth, flowering and yield of African marigold (Tagetes erecta Linn.) cv. 'Siracole'. Indian J. Natural Products and Resources, 1 (3), 371-375.

Pushkar, N.C. and Singh, A.K. (2012) Effect of pinching and growth retardants on yield of African marigold (Tagetes erecta L.) var. Pusa Narangi Gainda. Inter. J. of Hort., 2 (1), 1-4.

Rivas-San V.M. and Plasencia, J. (2011) Salicylic acid beyond defence: its role in plant growth and development. J. Exp. Bot., 62, 3321-3338.

Razmjoo, S. (1997) "Manual of Analysis of Fruit and Vegetable Products", $9^{\text {th }}$ ed. Tata Mc Graw Hill, New Delhi.

Ryagi, V.Y., Mantur, S.M. and Reddy, B.S. (2007) Effect of pinching on growth, yield and quality of flowers of Carnation variety grown under polyhouse. Karnataka J. of Agric. Sci., 20 (4), 816-818.

Saric, M., Curic, R., Cupina, T. and Geric, I. (1976) Chlorophyll Determination. "Univerzit eU Novon Sadu". Praktikum iz Fiziologize Biljaka-Beograd, Haucna Anjiga p 215.

Shakirova, F.E., Sakhabutdinova, A.R., Bezrukova, M.V., Fatkhutdinova, R.A. and Fatkhutdinova, D.R. (2003) Changes in the hormonal status of wheat seedlings induced by Salicylic acid and salinity. Plant Sci., 164, 317-322 .

Singh, M.R.S., Ganesha, R. and Ramesh, S. (2005) Effects of nitrogen, phosphorous and potassium herbage, oil yield, oil quality and soil fertility status of lemongrass in a semi-arid tropical region of India, J. Hortic. Sci. Biotech., 80, 493-497.

Singh, M.R.S., Ganesha, R. and Ramesh, S. (2007) Effects of N and K on growth, herbage, oil yield and nutrient uptake patterns of rosemary under semi-arid tropical conditions, J. Hortic. Sci. Biotech., 82, 414-419.

Winter-Sluter, A. and Kramer, D. (1977) Cytochemical localization of $\mathrm{K}^{+}$stimulated adenosine triphosphatase activity. Plant Physiol., 60 (6), 923-927.

Yassen, A.A., Habib, A.M., Zaghloul, S.M. and Khaled, S.M. (2010) Effect of different sources of potassium fertilizers on growth yield and chemical composition of Calendula officinalis. J. Amer. Sci., 6 (12), $1044-11048$. 


\section{تأثثر إزالة القمة النامية ومصادر البوتاسيوم علي النمو ومحصول

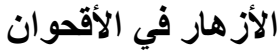

$$
\begin{aligned}
& \text { سهام محمد عبد الحميد الجمل } \\
& \text { قسم بحوث النباتات الطبية و العطرية - معهز بحوث البساتين - مركز البحوث } \\
& \text { الزر اعية ـ القاهرة - مصر . }
\end{aligned}
$$

تهذف هذه الدراسة إلي زيادة الألون الطبيعية في نبات الأقحوان باستخدام الطرق

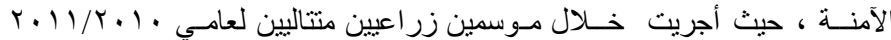

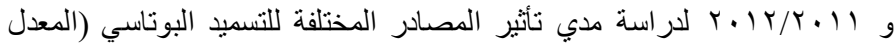

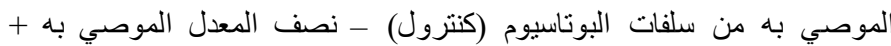

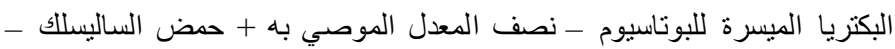

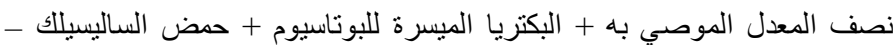

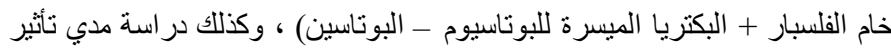

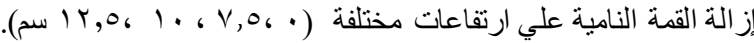

أظهرت النتائج التأثير الإيجابي لمصادر التسميد البوناسي المختلفة في زيادة

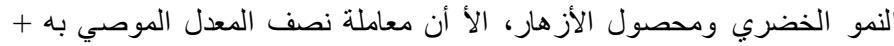

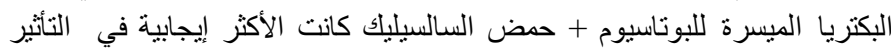

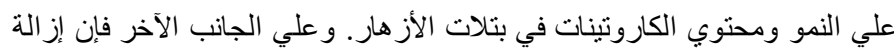

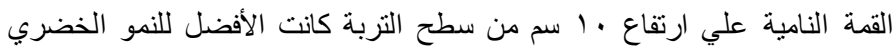

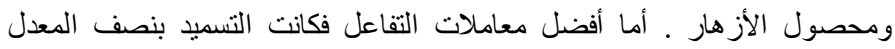

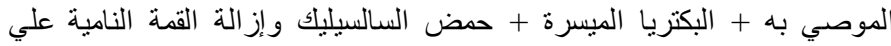

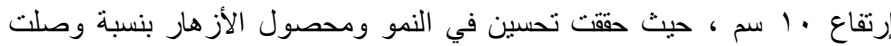

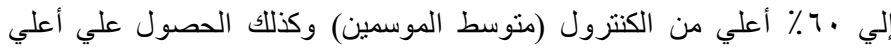

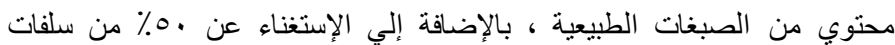
البوتاسيوم وتلافي أثرها الضار علي الإنسان و البيئة كليهما. الكلمات الرئيسية : الأقحوان ، البوتاسيوم ، إز الة القمة النامية ، الكاروتينات . 